\title{
Properties of standing Kruskal-Schwarzschild-modes at the magnetopause
}

\author{
F. Plaschke ${ }^{1}$ and K.-H. Glassmeier ${ }^{1,2}$ \\ ${ }^{1}$ Institut für Geophysik und extraterrestrische Physik, Technische Universität Braunschweig, Germany \\ ${ }^{2}$ Max-Planck-Institut für Sonnensystemforschung, Katlenburg-Lindau, Germany
}

Received: 19 May 2011 - Revised: 22 August 2011 - Accepted: 4 October 2011 - Published: 14 October 2011

\begin{abstract}
The radial, oscillatory motion of the Earth's magnetopause has been found to occur predominantly with some distinct, sometimes called "magic" frequencies, which have been attributed to magnetospheric wave guide modes, typical solar wind variations or, more recently, surface waves on the magnetopause standing between the northern and southern ionospheres. In this paper we present for the first time a derivation of these surface waves, denominated as KruskalSchwarzschild-modes (KS-modes), in the approximation of the ideal, single-fluid magnetohydrodynamic theory for incompressible plasmas. The calculations are performed in the simplified geometry of the box magnetosphere with the magnetopause being a plane between two plasma regimes of homogeneous conditions. The reflection of the KS-modes at the ionospheres is being discussed. Under the given assumptions and realistic conditions the validity of the calculations is shown to be limited to cases of parallel or anti-parallel background magnetic fields on both sides of the magnetopause, respectively. For these cases a detailed discussion of the mode structure is presented. The magnetopause when affected by a KS-mode is found to resemble a membrane under tension with respect to its motion; the ionospheres act as supporting points of the membrane and the KS-modes correspond in this picture to their eigenmodes of oscillation. Localized pressure enhancements in the magnetosheath are discussed as possible excitation mechanism for the KS-modes.
\end{abstract}

Keywords. Magnetospheric physics (Magnetopause, cusp, and boundary layers; Magnetosphere-ionosphere interactions; MHD waves and instabilities)

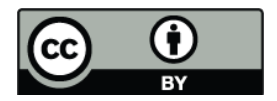

Correspondence to: F. Plaschke (f.plaschke@tu-bs.de)

\section{Introduction}

The magnetopause represents the boundary which separates the magnetospheric plasma and magnetic field from their counterparts of solar origin in the magnetosheath. Usually, in the absence of reconnection, it can be treated as a tangential discontinuity, across which hardly any normal flow of plasma takes place (Sonnerup and Cahill, 1967; Sonnerup and Ledley, 1974). Although most of the plasma parameters change across the boundary (for instance direction and strength of the magnetic field, particle densities and temperatures), the magnetopause is characterized by an equilibrium of the total pressure (Paschmann et al., 1993; Phan and Paschmann, 1996): On the magnetosheath side the thermal and magnetic pressures may be of similar importance; they are balanced by the dominant magnetic pressure on the magnetospheric side where, nevertheless, the thermal pressure may also be non-negligible. Furthermore, the magnetic tension due to the curvature of the magnetic field contributes on both sides to the overall pressure balance, which determines the shape and (average) location of the magnetopause (Spreiter et al., 1966; Shue and Song, 2002).

Motion and undulation of the magnetopause have first been studied using single spacecraft measurements (e.g. Aubry et al., 1971). Its radial velocity and the thickness of its current and boundary layers could first be reliably determined with data from the two neighboring spacecraft ISEE 1 and 2 using timing techniques (Berchem and Russell, 1982; Le and Russell, 1994). These techniques became most useful when applied to measurements of the four spacecraft mission CLUSTER (Paschmann et al., 2005; Panov et al., 2008), which also opened the door to more sophisticated analysis methods and inter-method comparisons (Haaland et al., 2004). However, the reconstruction of the (radial) magnetopause motion over extended periods of time remained subject to the integration of the boundary normal plasma velocity

Published by Copernicus Publications on behalf of the European Geosciences Union. 


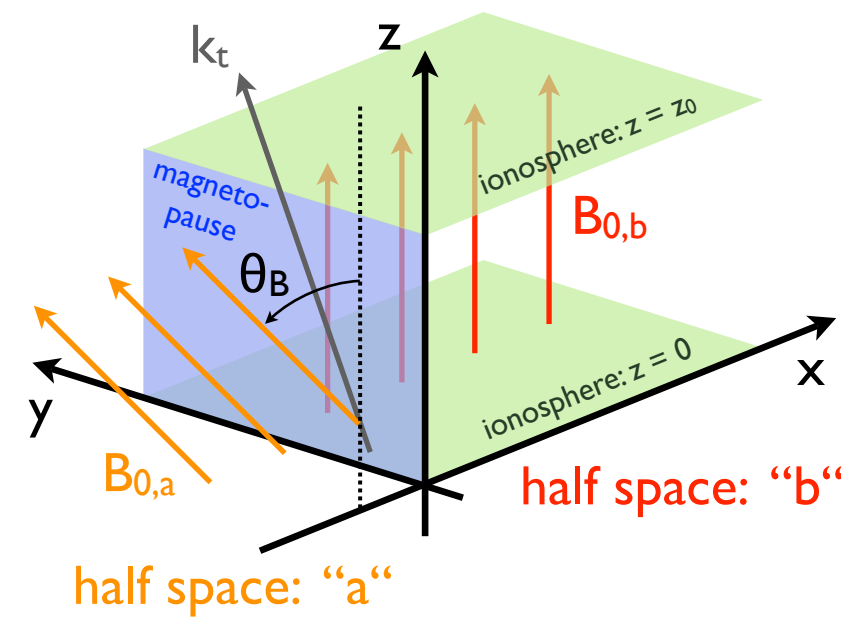

Fig. 1. Sketch of the box magnetosphere: Half space "b" (background) corresponds to the inner magnetosphere. Here the background magnetic field points in z-direction and is confined between the ionospheric boundaries (green surfaces) at $z=0$ (south) and $z=z_{0}$ (north). In the magnetosheath (half space "a", foreground) the background magnetic field may be tilted within the y-z-plane. The magnetopause is located between the half spaces at $x=0$ (blue surface).

measured in the vicinity of the magnetopause (Paschmann et al., 1990) or the more advanced empirical reconstruction method (De Keyser et al., 2005) until the start of the five spacecraft mission THEMIS (Angelopoulos, 2008).

During the first months of operation (coast-phase: February to September 2007) the five THEMIS spacecraft were flying in a row on a common coast-phase orbit. The scale size of this so-called string-of-pearls configuration allowed for a direct reconstruction of the magnetopause motion solely based on the observed magnetopause crossing times and corresponding positions of the respective spacecraft. Intervals between subsequent crossings were bridged using spline functions (Glassmeier et al., 2008). A statistical analysis of the coast-phase data with this method yielded distributions of the radial boundary velocity, amplitude, and most notably, frequency of oscillation (Plaschke et al., 2009a). Interestingly, the magnetopause was found to oscillate with higher probability of occurrence with some distinct frequencies around 1.3, 1.9, 2.7, 3.1 and $4.1 \mathrm{mHz}$ (Plaschke et al., 2009b).

Two decades ago the lowest three of these frequencies were found to stand out in ionospheric radar measurements of geomagnetic pulsations (Ruohoniemi et al., 1991; Samson et al., 1991). Due to their stability and recurrence they were attributed to magnetospheric cavity or wave guide modes (Samson et al., 1992) and called CMS frequencies after the cavity mode model of Samson. The distinctiveness of these frequencies has been supported by some studies of geomagnetic pulsations (e.g. Fenrich et al., 1995; Francia and Villante, 1997; Mathie et al., 1999) and questioned by others (e.g. Ziesolleck and McDiarmid, 1995). Sometimes the term "magic" has been used for their denomination, which may be more appropriate considering the lack of direct evidence for the existence of the cavity or wave guide modes (e.g. Sarafopoulos, 2005). Kepko et al. (2002) and Kepko and Spence (2003) proposed that the discrete "magic" frequencies (MF) could originate from typical length scales of density variations in the solar wind. In the Earth's frame of reference these length scales are converted into typical frequencies of geomagnetic pulsations driven by the corresponding solar wind variations.

A third explanation has been more recently suggested by Plaschke et al. (2009b), where the magnetopause takes a more active role: Surface waves on the magnetopause which are reflected at and standing between the ionospheric footpoints of closed field line shells may also account for the frequency selection observed. Surface waves propagating on the interface between two different plasma regimes have first been predicted by Kruskal and Schwarzschild (1954); as a tribute to their pioneering work they are denominated as Kruskal-Schwarzschild-modes (KS-modes).

Standing KS-modes on the magnetopause represent surface eigenmodes of the boundary. These modes should be damped in the presence of a gradual change between the plasma regimes (Chen and Hasegawa, 1974b; Uberoi, 1989) due to resonant absorption and excitation of shear Alfvén waves within the boundary (see also Belmont et al., 1995; De Keyser et al., 1999). Non-standing surface waves may also be generated by the Kelvin-Helmholtz instability (e.g. $\mathrm{Pu}$ and Kivelson, 1983; Fujita et al., 1996). The aim of this paper is to calculate and discuss the properties of the standing KS-modes in the context of the ideal, single-fluid magnetohydrodynamic (MHD) theory for incompressible plasmas. The reflection of the KS-modes at the ionosphere as well as their excitation are also being addressed.

\section{Basic equations}

The standing KS-modes on the magnetopause are derived in the simplified geometry of the box magnetosphere (e.g. Southwood, 1974). Figure 1 depicts the situation considered: The x-direction corresponds to the radially inward pointing direction in the equatorial magnetosphere. The magnetopause is located at $x=0$. Positive values of $x$ indicate a location inside the (inner) magnetosphere (half space "b"), negative values $(x<0)$ correspond to the magnetosheath half space "a". In this geometry the background magnetic field is stratified and homogeneous in both half spaces; it points in the z-direction in the magnetosphere: $\boldsymbol{B}_{0, \mathrm{~b}}=B_{0, \mathrm{~b}} \boldsymbol{e}_{\mathrm{Z}}$, where $\boldsymbol{e}_{\mathrm{z}}$ denotes the unit vector in the $\mathrm{z}$ direction. The direction of the background magnetic field in the magnetosheath (half space "a") shall only be restricted to planes parallel to the magnetopause (y-z-plane): $\boldsymbol{B}_{0, \mathrm{a}}=B_{0, \mathrm{a}}\left(0, \sin \theta_{B}, \cos \theta_{B}\right)^{\mathrm{T}}$. Since the background fields are tangential to the magnetopause in both half spaces, 
the boundary in between can be regarded as a tangential discontinuity.

The magnetospheric magnetic field is delimited in the $\mathrm{z}$ direction by the northern and southern ionospheres; in the box magnetosphere these correspond to x-y-planes at $z=0$ (south) and $z=z_{0}$ (north). The ionospheres are assumed to be infinitely conducting layers at which the electric field vanishes. The magnetosheath magnetic field, instead, is not bounded by the ionospheres. The box magnetosphere model can only be a rough approximation to reality. In particular the geometry of the cusp region is extremely simplified: close to the ionosphere magnetospheric and magnetosheath flux tubes should no longer be adjacent to each other; their separation, however, cannot be reflected in the model due to the stratification of the magnetic fields.

For the derivation of the basic equations we follow the computations detailed in De Keyser et al. (1999): The governing equations in each of the two half spaces shall be the ideal, single-fluid MHD equations:

$$
\begin{aligned}
\rho\left(\frac{\partial \boldsymbol{v}}{\partial t}+(\boldsymbol{v} \cdot \nabla) \boldsymbol{v}\right) & =-\nabla p-\nabla \frac{B^{2}}{2 \mu_{0}}+\frac{1}{\mu_{0}}(\boldsymbol{B} \cdot \nabla) \boldsymbol{B} \\
\frac{\partial \rho}{\partial t} & =-\nabla \cdot(\rho \boldsymbol{v}) \\
\frac{\partial \boldsymbol{B}}{\partial t} & =-\nabla \times \boldsymbol{E} \\
\boldsymbol{E} & =-\boldsymbol{v} \times \boldsymbol{B}
\end{aligned}
$$

Here $\rho$ denotes the mass density, $\boldsymbol{v}$ the plasma bulk velocity, $p$ the isotropic thermal pressure, $B=|\boldsymbol{B}|$ the modulus of the magnetic field and $\boldsymbol{E}$ the electric field. As usual, the vacuum permeability constant is given by $\mu_{0} ; t$ denotes the time. Equation (4), the generalized Ohm's law for the ideally conducting plasma, can be used to substitute $\boldsymbol{E}$ in the induction Eq. (3). The derivative $\partial \rho / \partial t$ may be replaced by $C_{\mathrm{S}}^{-2} \partial p / \partial t$, where $C_{\mathrm{S}}$ denotes the sound speed. A plane wave ansatz

$Q=Q_{0}+\delta Q=Q_{0}+\tilde{Q} e^{i \boldsymbol{k} \cdot \boldsymbol{r}-i \omega t}$

is adopted for the quantities $\rho, p, \boldsymbol{v}, \boldsymbol{B}$ and $\boldsymbol{E}$; here $\boldsymbol{k}=$ $\left(k_{\mathrm{x}}, k_{\mathrm{y}}, k_{\mathrm{z}}\right)^{\mathrm{T}}$ is the wave vector of the perturbations considered and $\omega$ the respective frequency. A vanishing background electric field and velocity $\left(\boldsymbol{E}_{0}=0\right.$ and $\left.\boldsymbol{v}_{0}=0\right)$ as well as constant background density $\left(\rho_{0}\right)$, pressure $\left(p_{0}\right)$ and magnetic field $\left(\boldsymbol{B}_{0}\right)$ are assumed in either of the two half spaces. The amplitudes of the perturbations in these quantities, denoted with a tilde sign according with Eq. (5), are also taken as constant such that temporal and spatial variations are only given by the exponential functions. Under these assumptions the Eqs. (1) to (3) can be rewritten after linearization as:

$$
\begin{aligned}
\omega \rho_{0} \tilde{\boldsymbol{v}} & =\boldsymbol{k} \tilde{\tau}-\left(\boldsymbol{k} \cdot \boldsymbol{B}_{0}\right) \frac{\tilde{\boldsymbol{B}}}{\mu_{0}} \\
\omega \tilde{\boldsymbol{p}} & =(\boldsymbol{k} \cdot \tilde{\boldsymbol{v}}) C_{\mathrm{S}}^{2} \rho_{0} \\
\omega \tilde{\boldsymbol{B}} & =-\left(\boldsymbol{k} \cdot \boldsymbol{B}_{0}\right) \tilde{\boldsymbol{v}}+(\boldsymbol{k} \cdot \tilde{\boldsymbol{v}}) \boldsymbol{B}_{0}
\end{aligned}
$$

Here $\tilde{\tau}$ denotes the total pressure perturbation (e.g. De Keyser et al., 1999):

$\tilde{\tau}=\tilde{p}+\frac{\boldsymbol{B}_{0} \cdot \tilde{\boldsymbol{B}}}{\mu_{0}}$

After replacing $\tilde{p}$ in Eq. (7) with $\tilde{\tau}$ from Eq. (9) we obtain a system of seven scalar equations for the perturbed quantities $\tilde{\boldsymbol{v}}, \tilde{\boldsymbol{B}}$ and $\tilde{\tau}$. Elimination of all of these quantities yields the well-known dispersion relations for the three MHD wave modes (e.g. De Keyser et al., 1999):

$\Omega^{2}:=\omega^{2}-\left(\boldsymbol{k} \cdot \boldsymbol{V}_{\mathrm{A}}\right)^{2}=0$

$k_{\mathrm{x}}^{2}=\frac{\omega^{2}}{V_{\mathrm{A}}^{2}+C_{\mathrm{S}}^{2} \Omega^{2} / \omega^{2}}-k_{\mathrm{t}}^{2}$

where $\boldsymbol{V}_{\mathrm{A}}=\boldsymbol{B}_{0} / \sqrt{\mu_{0} \rho_{0}}$ is the Alfvén velocity and $\boldsymbol{k}_{\mathrm{t}}=$ $\left(0, k_{\mathrm{y}}, k_{\mathrm{z}}\right)^{\mathrm{T}}$ denotes the projection of the wave vector $\boldsymbol{k}$ onto the magnetopause plane. Equation (10) is the dispersion relation for the shear Alfvén wave, for which $\tilde{\tau}=0$ holds. Equation (11) contains the dispersion relations for both magnetosonic waves (fast and slow mode), where $\tilde{\tau}$ shall be nonvanishing.

The KS-modes on the magnetopause considered in this paper consist of two evanescent magnetosonic surface waves, one in each of the two half spaces, for which Eq. (11) holds. Their amplitude shall decay exponentially with increasing distance from the magnetopause: Hence, $k_{\mathrm{x}}^{2}$ has to be negative on both sides of the boundary.

Following Walker (1981) the boundary normal component of the velocity $\tilde{v}_{\mathrm{X}}$ and the pressure perturbation $\tilde{\tau}$ need to be continuous at the magnetopause $(x=0)$ :

$$
\begin{aligned}
\tilde{\tau}_{\mathrm{a}} & =\tilde{\tau}_{\mathrm{b}} \\
\tilde{v}_{\mathrm{x}, \mathrm{a}} & =\tilde{v}_{\mathrm{x}, \mathrm{b}}
\end{aligned}
$$

such that pressure equilibrium is maintained at all times. The indices "a" and "b" denote here the half space, to which the respective quantities pertain. Furthermore, the frequency of the perturbations $\omega$ and the boundary tangential wave vector $\boldsymbol{k}_{\mathrm{t}}$ are also equal everywhere. The standing KS-modes have to satisfy the boundary conditions (12) and (13), from which their dispersion relation can be computed. For this purpose a relation between $\tilde{v}_{\mathrm{x}}$ and $\tilde{\tau}$ is needed, which can be obtained from Eq. (6); substitution of $\tilde{\boldsymbol{B}}$ with Eq. (8) yields:

$\tilde{\boldsymbol{v}} \Omega^{2}=\frac{\omega \tilde{\tau}}{\rho_{0}} \boldsymbol{k}-\left(\boldsymbol{k} \cdot \boldsymbol{V}_{\mathrm{A}}\right)(\boldsymbol{k} \cdot \tilde{\boldsymbol{v}}) \boldsymbol{V}_{\mathrm{A}}$

Since $V_{\mathrm{A}}$ is restricted to y-z-planes in both half spaces, the $\mathrm{x}$-component of Eq. (14) is:

$\tilde{v}_{\mathrm{X}} \Omega^{2}=\omega k_{\mathrm{X}} \tilde{\tau} / \rho_{0}$

Herewith the Eqs. (12) and (13) can be combined to obtain the general dispersion relation for the magnetopause surface waves or KS-modes (e.g. Pu and Kivelson, 1983):

$\frac{k_{\mathrm{x}, \mathrm{a}}}{\rho_{0, \mathrm{a}} \Omega_{\mathrm{a}}^{2}}=\frac{k_{\mathrm{x}, \mathrm{b}}}{\rho_{0, \mathrm{~b}} \Omega_{\mathrm{b}}^{2}}$ 
As already mentioned, the amplitude of the surface waves should decay exponentially with increasing distance from the boundary on either side of it: $k_{\mathrm{x}, \mathrm{a}}$ and $k_{\mathrm{x}, \mathrm{b}}$ have to have opposite signs, and $\Omega^{2}$ has to change sign at the boundary, too. Thus, any KS-mode on the magnetopause will propagate faster than an Alfvén wave in field parallel direction in one of the half spaces and slower in the other.

Simplification of the equations can be achieved, if the plasma is assumed to be incompressible $(\nabla \cdot v=0)$. Using the plane wave ansatz for $v$ we obtain the algebraic incompressibility condition: $\boldsymbol{k} \cdot \tilde{\boldsymbol{v}}=0$. Herewith, the rightmost term of Eq. (14) can be omitted yielding: $\tilde{\boldsymbol{v}} \sim \boldsymbol{k}$. Combining this relation with $\boldsymbol{k} \cdot \tilde{\boldsymbol{v}}=0$ we obtain:

$k^{2}=k_{\mathrm{x}}^{2}+k_{\mathrm{y}}^{2}+k_{\mathrm{z}}^{2}=0$

This relation substitutes the dispersion relation (11) in the case of incompressibility; it can also be obtained from that equation under the assumption $C_{\mathrm{S}}=\infty$, which is equivalent to the incompressibility condition.

It should be noted that the assumption of incompressibility on both sides of the magnetopause is certainly not always justified. As will be demonstrated in Sect. 4 where the validity of the presented calculations is considered in detail, particularly the plasma on the magnetosheath side may have to be regarded as compressible. However, if the boundary tangential wave numbers $\boldsymbol{k}_{\mathrm{t}}$ are large enough with respect to the $\omega$-dependent term in Eq. (11), then this equation should be well approximated by Eq. (17). For these cases the assumption of incompressibility significantly simplifies the analytical treatment of the standing KS-modes, which is the aim of this paper. Otherwise the full dispersion relation (11) would have to be considered: Replacing $k_{\mathrm{x}, \mathrm{a}}$ and $k_{\mathrm{x}, \mathrm{b}}$ herewith in Eq. (16) yields a polynomial of degree 10 in $\omega$ for which no analytical solution can be found in general ( $\mathrm{Pu}$ and Kivelson, 1983).

If Eq. (17) holds, $k_{\mathrm{x}}^{2}<0$ is automatically enforced if $k_{\mathrm{y}}$ and $k_{\mathrm{z}}$ are chosen to be real numbers. Thus, surface waves occur at the magnetopause in the incompressible case regardless of the plasma background parameters in the two half spaces (Uberoi, 1989). Furthermore, $\left|k_{\mathrm{x}}\right|$ is independent from the side of the magnetopause. If compressibility were assumed (Eq. 11) $\left|k_{\mathrm{x}}\right|$ would be dependent on the plasma conditions of each side and, hence, would be different in both half spaces. $k_{\mathrm{x}}^{2}$ could also be positive corresponding to propagating fast or slow waves instead of evanescent surface waves.

The geometry of the box magnetosphere can be used to further simplify the dispersion relation (16). The background magnetic field in half space " $\mathrm{b}$ " has been assumed to point in the z-direction; it follows: $\Omega_{\mathrm{b}}^{2}=\omega^{2}-k_{\mathrm{z}}^{2} V_{\mathrm{A}, \mathrm{b}}^{2}$. The corresponding expression for half space "a" is: $\Omega_{\mathrm{a}}^{2}=\omega^{2}-$ $V_{\mathrm{A}, \mathrm{a}}^{2}\left(k_{\mathrm{y}} \sin \theta_{B}+k_{\mathrm{z}} \cos \theta_{B}\right)^{2}$. Substitution of $k_{\mathrm{x}, \mathrm{a}}=-i \sqrt{k_{\mathrm{t}}^{2}}$ and $k_{\mathrm{x}, \mathrm{b}}=i \sqrt{k_{\mathrm{t}}^{2}}$ via Eq. (17) finally yields the following equivalent relations:

$$
\begin{aligned}
\rho_{0, \mathrm{a}} \Omega_{\mathrm{a}}^{2} & =-\rho_{0, \mathrm{~b}} \Omega_{\mathrm{b}}^{2} \\
\omega^{2} & =\frac{\rho_{0, \mathrm{~b}} V_{\mathrm{A}, \mathrm{b}}^{2} k_{\mathrm{z}}^{2}+\rho_{0, \mathrm{a}} V_{\mathrm{A}, \mathrm{a}}^{2}\left(k_{\mathrm{y}} \sin \theta_{B}+k_{\mathrm{z}} \cos \theta_{B}\right)^{2}}{\rho_{0, \mathrm{~b}}+\rho_{0, \mathrm{a}}}
\end{aligned}
$$

As mentioned above, these equations can also be derived for the compressible case by substituting $k_{\mathrm{x}}$ in Eq. (16) with relation (11).

\section{Standing Kruskal-Schwarzschild-modes}

The above given equations constitute the basis for studying the polarization properties of standing KS-modes. We start with the electric field given by Eq. (4) in half space "b" (magnetosphere): $\tilde{\boldsymbol{E}}_{\mathrm{b}}=-\tilde{\boldsymbol{v}}_{\mathrm{b}} \times \boldsymbol{B}_{0, \mathrm{~b}}$. Replacing $\tilde{\boldsymbol{v}}$ with Eq. (14) for the incompressible case $(\boldsymbol{k} \cdot \tilde{\boldsymbol{v}}=0)$ yields:

$$
\begin{aligned}
\tilde{\boldsymbol{E}}_{\mathrm{b}} & =-\frac{\omega \tilde{\tau}}{\Omega_{\mathrm{b}}^{2} \rho_{0, \mathrm{~b}}} \boldsymbol{k} \times \boldsymbol{B}_{0, \mathrm{~b}} \\
& =-\frac{\omega \tilde{\tau}}{\Omega_{\mathrm{b}}^{2} \rho_{0, \mathrm{~b}}}\left(k_{\mathrm{y}} B_{0, \mathrm{~b}} \boldsymbol{e}_{\mathrm{x}}-k_{\mathrm{x}, \mathrm{b}} B_{0, \mathrm{~b}} \boldsymbol{e}_{\mathrm{y}}\right)
\end{aligned}
$$

At the ionospheres at $z=0$ and $z=z_{0}$ the electric field in the $x-y-p l a n e$ should vanish due to the high Pedersen conductivity, assumed to be infinite. An incident magnetopause surface wave will be reflected there; both, incident and reflected waves (upper indizes I and R, respectively) will be superposed such that the condition for the electric field is satisfied at the ionospheres $\left(\boldsymbol{E}_{\mathrm{b}}=\mathbf{0}\right)$. Hence, to find a solution in the specified geometry a second wave of equal amplitude with respect to the incident wave needs to be added, which propagates in opposite direction $(-\mathrm{z})$ with a phase shift of $180^{\circ}$.

Since perfect reflection of the incident wave is assumed, the ionospheric dissipation of the wave's energy due to Joule heating and the consequent damping are neglected. Furthermore, the Hall conductivity shall not be considered. This implies that additional ionospheric evanescent waves (Allan and Knox, 1979, 1982) generated by Hall currents upon reflection of an incident magnetopause surface wave are also neglected.

For the reflected wave $k_{\mathrm{x}, \mathrm{b}}, k_{\mathrm{y}}$ and $\omega$ will remain unchanged. It follows from the incompressibility condition (17), that $k_{\mathrm{z}}^{2}$ should be equal, too. Only the sign of $k_{\mathrm{z}}$ can and must differ. Since the plasma parameters $B_{0, \mathrm{~b}}$ and $\rho_{0, \mathrm{~b}}$ also hold for both waves, the relations $\tilde{E}_{\mathrm{x}, \mathrm{b}}^{\mathrm{I}}=-\tilde{E}_{\mathrm{x}, \mathrm{b}}^{\mathrm{R}}$ and $\tilde{E}_{\mathrm{y}, \mathrm{b}}^{\mathrm{I}}=-\tilde{E}_{\mathrm{y}, \mathrm{b}}^{\mathrm{R}}$ can only be satisfied in:

$$
\begin{aligned}
\delta \boldsymbol{E}_{\mathrm{b}} & =\left(\tilde{E}_{\mathrm{x}, \mathrm{b}}^{\mathrm{I}}, \tilde{E}_{\mathrm{y}, \mathrm{b}}^{\mathrm{I}}, 0\right)^{\mathrm{T}} e^{i\left(k_{\mathrm{x}, \mathrm{b}} x+k_{\mathrm{y}} y-\omega t\right)} e^{i k_{\mathrm{z}} z} \\
& +\left(\tilde{E}_{\mathrm{x}, \mathrm{b}}^{\mathrm{R}}, \tilde{E}_{\mathrm{y}, \mathrm{b}}^{\mathrm{R}}, 0\right)^{\mathrm{T}} e^{i\left(k_{\mathrm{x}, \mathrm{b}} x+k_{\mathrm{y}} y-\omega t\right)} e^{-i k_{\mathrm{z}} z}
\end{aligned}
$$


with $k_{\mathrm{z}}:=k_{\mathrm{z}}^{\mathrm{I}}=-k_{\mathrm{z}}^{\mathrm{R}}$ and

$\left(\tilde{E}_{\mathrm{x}, \mathrm{b}}^{\mathrm{I}}, \tilde{E}_{\mathrm{y}, \mathrm{b}}^{\mathrm{I}}, 0\right)^{\mathrm{T}}=\frac{\omega \tilde{\tau}^{\mathrm{I}} B_{0, \mathrm{~b}}}{\left(\Omega_{\mathrm{b}}^{\mathrm{I}}\right)^{2} \rho_{0, \mathrm{~b}}}\left(-k_{\mathrm{y}}, k_{\mathrm{x}, \mathrm{b}}, 0\right)^{\mathrm{T}}$

$\left(\tilde{E}_{\mathrm{x}, \mathrm{b}}^{\mathrm{R}}, \tilde{E}_{\mathrm{y}, \mathrm{b}}^{\mathrm{R}}, 0\right)^{\mathrm{T}}=\frac{\omega \tilde{\tau}^{\mathrm{R}} B_{0, \mathrm{~b}}}{\left(\Omega_{\mathrm{b}}^{\mathrm{R}}\right)^{2} \rho_{0, \mathrm{~b}}}\left(-k_{\mathrm{y}}, k_{\mathrm{x}, \mathrm{b}}, 0\right)^{\mathrm{T}}$

from Eq. (19), if the quotients of $\tilde{\tau}$ and $\Omega_{\mathrm{b}}^{2}$ in the Eqs. (21) and (22) differ only by their sign:

$$
\frac{\tilde{\tau}^{\mathrm{I}}}{\left(\Omega_{\mathrm{b}}^{\mathrm{I}}\right)^{2}}=-\frac{\tilde{\tau}^{\mathrm{R}}}{\left(\Omega_{\mathrm{b}}^{\mathrm{R}}\right)^{2}}=: \frac{\tilde{\tau}}{\Omega_{\mathrm{b}}^{2}}
$$

Taking this into account the disturbance of the electric field (Eq. 20) can be rewritten as:

$\delta \boldsymbol{E}_{\mathrm{b}}=2 i\left(\tilde{E}_{\mathrm{x}, \mathrm{b}}^{\mathrm{I}}, \tilde{E}_{\mathrm{y}, \mathrm{b}}^{\mathrm{I}}, 0\right)^{\mathrm{T}} e^{i\left(k_{\mathrm{x}, \mathrm{b}} x+k_{\mathrm{y}} y-\omega t\right)} \sin \left(k_{\mathrm{z}} z\right)$

The factor $\sin \left(k_{\mathrm{z}} z\right)$ is inherent in Eq. (20) and can be obtained using Eq. (23):

$P_{+, \mathrm{b}}:=\frac{\tilde{\tau}^{\mathrm{I}} e^{i k_{\mathrm{z}} z}}{\left(\Omega_{\mathrm{b}}^{\mathrm{I}}\right)^{2}}+\frac{\tilde{\tau}^{\mathrm{R}} e^{-i k_{\mathrm{z}} z}}{\left(\Omega_{\mathrm{b}}^{\mathrm{R}}\right)^{2}}=2 i \frac{\tilde{\tau}}{\Omega_{\mathrm{b}}^{2}} \sin \left(k_{\mathrm{z}} z\right)$

It should be noted that a minus sign in this sum would result in a factor $\cos \left(k_{\mathrm{z}} z\right)$. More generally, we define $P_{ \pm, \mathrm{a} / \mathrm{b}}$ as follows to simplify some equations in this section:

$P_{ \pm, \mathrm{a} / \mathrm{b}}:=\frac{\tilde{\tau}^{\mathrm{I}} e^{i k_{\mathrm{z}} z}}{\left(\Omega_{\mathrm{a} / \mathrm{b}}^{\mathrm{I}}\right)^{2}} \pm \frac{\tilde{\tau}^{\mathrm{R}} e^{-i k_{\mathrm{z}} z}}{\left(\Omega_{\mathrm{a} / \mathrm{b}}^{\mathrm{R}}\right)^{2}}$

As can be seen, the tangential electric field at the ionospheres (at $z=0$ and $z=z_{0}$ ) vanishes, if the condition

$k_{\mathrm{z}} z_{0}=n \pi \quad$ for $\quad n \in \mathbb{N}$

is satisfied. Hence, for a KS-mode to be standing on the magnetopause a multiple of half its wave length in the $\mathrm{z}$-direction $(\lambda / 2)$ must fit between the ionospheres: $z_{0}=n \lambda / 2$. This discretization of $\lambda$ directly results from the confinement of the background magnetic field in the magnetosphere between the totally reflecting ionospheric boundaries.

From the x- and y-components of the ansatz (20) of $\delta \boldsymbol{E}_{\mathrm{b}}$ the corresponding velocity components can be easily calculated via the linearized Eq. (4):

$$
\begin{aligned}
\left(\delta v_{\mathrm{x}, \mathrm{b}}, \delta v_{\mathrm{y}, \mathrm{b}}\right) & =\left(\delta E_{\mathrm{y}, \mathrm{b}},-\delta E_{\mathrm{x}, \mathrm{b}}\right) / B_{0, \mathrm{~b}} \\
= & \left(k_{\mathrm{x}, \mathrm{b}}, k_{\mathrm{y}}\right) \frac{\omega}{\rho_{0, \mathrm{~b}}} e^{i\left(k_{\mathrm{x}, \mathrm{b}} x+k_{\mathrm{y}} y-\omega t\right)} P_{+, \mathrm{b}} \\
= & 2 i\left(k_{\mathrm{x}, \mathrm{b}}, k_{\mathrm{y}}\right) \frac{\omega \tilde{\tau}}{\Omega_{\mathrm{b}}^{2} \rho_{0, \mathrm{~b}}} \cdot \\
& \cdot e^{i\left(k_{\mathrm{x}, \mathrm{b}} x+k_{\mathrm{y}} y-\omega t\right)} \sin \left(k_{\mathrm{z}} z\right)
\end{aligned}
$$

The z-component of the velocity field can be obtained via the incompressibility condition $\left(\nabla \cdot \delta \boldsymbol{v}_{\mathrm{b}}=0\right)$ :

$$
\frac{\partial}{\partial z} \delta v_{\mathrm{z}, \mathrm{b}}=-\frac{\partial}{\partial x} \delta v_{\mathrm{x}, \mathrm{b}}-\frac{\partial}{\partial y} \delta v_{\mathrm{y}, \mathrm{b}}
$$

Derivation of the components of Eq. (28) and subsequent integration of Eq. (30) yields after replacing $k_{\mathrm{x}, \mathrm{b}}^{2}+k_{\mathrm{y}}^{2}$ by $-k_{\mathrm{z}}^{2}$ via Eq. (17):

$\delta v_{\mathrm{z}, \mathrm{b}}=k_{\mathrm{z}} \frac{\omega}{\rho_{0, \mathrm{~b}}} e^{i\left(k_{\mathrm{x}, \mathrm{b}} x+k_{\mathrm{y}} y-\omega t\right)} P_{-, \mathrm{b}}$

Finally we obtain for the velocity disturbance of the standing KS-mode in half space "b":

$\delta \boldsymbol{v}_{\mathrm{b}}=\left(\begin{array}{c}i k_{\mathrm{x}, \mathrm{b}} \sin \left(k_{\mathrm{z}} z\right) \\ i k_{\mathrm{y}} \sin \left(k_{\mathrm{z}} z\right) \\ k_{\mathrm{z}} \cos \left(k_{\mathrm{z}} z\right)\end{array}\right) \frac{2 \omega \tilde{\tau}}{\Omega_{\mathrm{b}}^{2} \rho_{0, \mathrm{~b}}} e^{i\left(k_{\mathrm{x}, \mathrm{b}} x+k_{\mathrm{y}} y-\omega t\right)}$

It should be noted, that the amplitudes of the two superposed waves contributing to the disturbance of the velocity field are consistent with Eq. (14) for the incompressible case, although they have not been directly obtained using this equation.

From Eq. (32) the disturbance of the magnetic field can be easily determined using the induction Eq. (3). Replacement of $\boldsymbol{E}$ with Eq. (4), usage of $\boldsymbol{B}_{0, \mathrm{~b}}=B_{0, \mathrm{~b}} \boldsymbol{e}_{\mathrm{z}}$ as well as $\nabla \cdot \boldsymbol{B}=$ $0, \nabla \cdot \boldsymbol{v}=0$ and linearization yield:

$\frac{\partial}{\partial t} \delta \boldsymbol{B}_{\mathrm{b}}=\left(\boldsymbol{B}_{0, \mathrm{~b}} \cdot \nabla\right) \delta \boldsymbol{v}_{\mathrm{b}}=B_{0, \mathrm{~b}} \frac{\partial}{\partial z} \delta \boldsymbol{v}_{\mathrm{b}}$

With Eqs. (28) and (31) this results into:

$$
\begin{aligned}
\delta \boldsymbol{B}_{\mathrm{b}} & =-\frac{B_{0, \mathrm{~b}}}{\rho_{0, \mathrm{~b}}} e^{i\left(k_{\mathrm{x}, \mathrm{b}} x+k_{\mathrm{y}} y-\omega t\right)}\left(\begin{array}{c}
k_{\mathrm{x}, \mathrm{b}} k_{\mathrm{z}} P_{-, \mathrm{b}} \\
k_{\mathrm{y}} k_{\mathrm{z}} P_{-, \mathrm{b}} \\
k_{\mathrm{z}}^{2} P_{+, \mathrm{b}}
\end{array}\right) \\
& =-\frac{2 B_{0, \mathrm{~b}} \tilde{\tau}}{\rho_{0, \mathrm{~b}} \Omega_{\mathrm{b}}^{2}} e^{i\left(k_{\mathrm{x}, \mathrm{b}} x+k_{\mathrm{y}} y-\omega t\right)}\left(\begin{array}{c}
k_{\mathrm{x}, \mathrm{b}} k_{\mathrm{z}} \cos \left(k_{\mathrm{z}} z\right) \\
k_{\mathrm{y}} k_{\mathrm{z}} \cos \left(k_{\mathrm{z}} z\right) \\
i k_{\mathrm{z}}^{2} \sin \left(k_{\mathrm{z}} z\right)
\end{array}\right)
\end{aligned}
$$

The quantities in half space "a" (magnetosheath) can be derived using the magnetopause boundary conditions (12) and (13). Both waves considered in half space " $b$ " (magnetosphere) necessarily have to have their counterparts in the magnetosheath; the spatial structure of these waves resulting from their ionospheric reflection on the magnetospheric side is imposed via the magnetopause boundary conditions to the waves in the magnetosheath, which lacks ionospheric reflecting boundaries.

In this half space " $a$ " the $\mathrm{x}$-component of the velocity field is given by:

$\delta v_{\mathrm{x}, \mathrm{a}}=\frac{\omega k_{\mathrm{x}, \mathrm{a}}}{\rho_{0, \mathrm{a}}} P_{+, \mathrm{a}} e^{i\left(k_{\mathrm{x}, \mathrm{a}} x+k_{\mathrm{y}} y-\omega t\right)}$

This equation satisfies $\delta v_{\mathrm{x}, \mathrm{a}}=\delta v_{\mathrm{x}, \mathrm{b}}$ at $x=0$ (boundary condition 13), if the following relation holds:

$\frac{k_{\mathrm{x}, \mathrm{a}} P_{+, \mathrm{a}}}{\rho_{0, \mathrm{a}}}=\frac{k_{\mathrm{x}, \mathrm{b}} P_{+, \mathrm{b}}}{\rho_{0, \mathrm{~b}}}$

Considering the continuity of $\tilde{\tau}$ at the magnetopause (boundary condition 12) Eq. (37) is satisfied, as it is equivalent to the sum of the dispersion relations (16) for each of the surface 
waves (indices I and R) composing the standing KS-mode. Furthermore, it can be easily seen, that Eq. (23) holds true also for half space "a".

The other two components of the velocity field can be obtained with Eq. (14) under the assumtion of an incompressible plasma, which $\delta \boldsymbol{v}_{\mathrm{b}}$ (Eq. 32) and $\delta v_{\mathrm{x}, \mathrm{a}}$ (Eq. 36) are consistent with. We obtain:

$$
\left(\delta v_{\mathrm{y}, \mathrm{a}}, \delta v_{\mathrm{z}, \mathrm{a}}\right)=\left(k_{\mathrm{y}} P_{+, \mathrm{a}}, k_{\mathrm{z}} P_{-, \mathrm{a}}\right) \frac{\omega}{\rho_{0, \mathrm{a}}} e^{i\left(k_{\mathrm{x}, \mathrm{a}} x+k_{\mathrm{y}} y-\omega t\right)}
$$

It shall be noted, that the sign in $P_{-, \mathrm{a}}$ is negative for $\delta v_{\mathrm{z}, \mathrm{a}}$, since $k_{\mathrm{z}}$ has opposite signs for the two considered waves. Altogether, a comparison of the velocity disturbances $\delta \boldsymbol{v}$ of both half spaces at the boundary $(x=0)$ yields, taking into account the dispersion relation (18), the boundary conditions (12) and (13) as well as the definition of $P$ (Eq. 26):

$$
\left(\begin{array}{l}
\delta v_{\mathrm{x}, \mathrm{a}} \\
\delta v_{\mathrm{y}, \mathrm{a}} \\
\delta v_{\mathrm{z}, \mathrm{a}}
\end{array}\right)=\left(\begin{array}{c}
\delta v_{\mathrm{x}, \mathrm{b}} \\
-\delta v_{\mathrm{y}, \mathrm{b}} \\
-\delta v_{\mathrm{z}, \mathrm{b}}
\end{array}\right)
$$

Hence, the plasma flow tangential to the magnetopause is expected to oscillate in opposite direction on both sides under the influence of a standing KS-mode.

Equations (36) and (38) can be further simplified with the help of Eq. (23):

$$
\delta \boldsymbol{v}_{\mathrm{a}}=\left(\begin{array}{c}
i k_{\mathrm{x}, \mathrm{a}} \sin \left(k_{\mathrm{z}} z\right) \\
i k_{\mathrm{y}} \sin \left(k_{\mathrm{z}} z\right) \\
k_{\mathrm{z}} \cos \left(k_{\mathrm{z}} z\right)
\end{array}\right) \frac{2 \omega \tilde{\tau}}{\Omega_{\mathrm{a}}^{2} \rho_{0, \mathrm{a}}} e^{i\left(k_{\mathrm{x}, \mathrm{a}} x+k_{\mathrm{y}} y-\omega t\right)}
$$

As can be seen, this relation is practically identical to the one of Eq. (32). The mentioned velocity shear across the magnetopause is consistent with the change in sign of $\rho_{0} \Omega^{2}$ (Eq. 18).

To obtain the disturbance of the magnetic field in half space "a" Eq. (33) has to be reevaluated for a non-vanishing $\mathrm{y}$-component of the background magnetic field $\boldsymbol{B}_{0, \mathrm{a}}$ :

$$
\begin{aligned}
\frac{\partial}{\partial t} \delta \boldsymbol{B}_{\mathrm{a}} & =\left(\boldsymbol{B}_{0, \mathrm{a}} \cdot \nabla\right) \delta \boldsymbol{v}_{\mathrm{a}} \\
& =B_{0, \mathrm{a}}\left(\sin \theta_{B} \frac{\partial}{\partial y} \delta \boldsymbol{v}_{\mathrm{a}}+\cos \theta_{B} \frac{\partial}{\partial z} \delta \boldsymbol{v}_{\mathrm{a}}\right)
\end{aligned}
$$

Herewith we obtain using Eqs. (36) and (38):

$$
\begin{aligned}
\delta \boldsymbol{B}_{\mathrm{a}}= & -\frac{B_{0, \mathrm{a}}}{\rho_{0, \mathrm{a}}} e^{i\left(k_{\mathrm{x}, \mathrm{a}} x+k_{\mathrm{y}} y-\omega t\right)} . \\
& \cdot\left\{k_{\mathrm{y}} \sin \theta_{B}\left(\begin{array}{c}
k_{\mathrm{x}, \mathrm{a}} P_{+, \mathrm{a}} \\
k_{\mathrm{y}} P_{+, \mathrm{a}} \\
k_{\mathrm{z}} P_{-, \mathrm{a}}
\end{array}\right)+k_{\mathrm{z}} \cos \theta_{B}\left(\begin{array}{c}
k_{\mathrm{x}, \mathrm{a}} P_{-, \mathrm{a}} \\
k_{\mathrm{y}} P_{-, \mathrm{a}} \\
k_{\mathrm{z}} P_{+, \mathrm{a}}
\end{array}\right)\right\} \\
= & -\frac{2 B_{0, \mathrm{a}} \tilde{\tau}}{\rho_{0, \mathrm{a}} \Omega_{\mathrm{a}}^{2}} e^{i\left(k_{\mathrm{x}, \mathrm{a}} x+k_{\mathrm{y}} y-\omega t\right)} . \\
& \cdot\left(\begin{array}{c}
i k_{\mathrm{x}, \mathrm{a}} k_{\mathrm{y}} \sin \theta_{B} \sin \left(k_{\mathrm{z}} z\right)+k_{\mathrm{x}, \mathrm{a}} k_{\mathrm{z}} \cos \theta_{B} \cos \left(k_{\mathrm{z}} z\right) \\
i k_{\mathrm{y}}^{2} \sin \theta_{B} \sin \left(k_{\mathrm{z}} z\right)+k_{\mathrm{y}} k_{\mathrm{z}} \cos \theta_{B} \cos \left(k_{\mathrm{z}} z\right) \\
k_{\mathrm{y}} k_{\mathrm{z}} \sin \theta_{B} \cos \left(k_{\mathrm{z}} z\right)+i k_{\mathrm{z}}^{2} \cos \theta_{B} \sin \left(k_{\mathrm{z}} z\right)
\end{array}\right)
\end{aligned}
$$

Ann. Geophys., 29, 1793-1807, 2011
From $\delta \boldsymbol{B}_{\mathrm{b}}$ (Eq. 35) and $\delta \boldsymbol{B}_{\mathrm{a}}$ (Eq. 43) the current densities in both half spaces $\left(\delta \boldsymbol{j}=\nabla \times \delta \boldsymbol{B} / \mu_{0}\right)$ can be easily computed:

$\delta \boldsymbol{j}_{\mathrm{a}}=\delta \boldsymbol{j}_{\mathrm{b}}=\mathbf{0}$

Hence, the KS-modes (surfaces waves) are not associated with any currents flowing inside of the two half spaces (magnetosphere and magnetosheath).

Currents do only flow on the magnetopause itself. These shall now be computed: At the boundary $(x=0)$ the surface current density $\boldsymbol{J}=\boldsymbol{J}_{0}+\delta \boldsymbol{J}$ is composed of the background value, associated with the background magnetic fields $\boldsymbol{B}_{0}$ in both half spaces, and of its disturbance $\delta \boldsymbol{J}$ given by $\delta \boldsymbol{B} . \boldsymbol{J}_{0}$ is the steady Chapman-Ferraro (CF) current on the magnetopause. The surface current can be computed from the curl of the magnetic field across the discontinuity (at $x=0$ ) using Stokes' theorem:

$\boldsymbol{J}=\left(\begin{array}{c}J_{\mathrm{y}} \\ J_{\mathrm{z}}\end{array}\right)=\frac{1}{\mu_{0}}\left(\begin{array}{c}B_{\mathrm{z}, \mathrm{a}}-B_{\mathrm{z}, \mathrm{b}} \\ B_{\mathrm{y}, \mathrm{b}}-B_{\mathrm{y}, \mathrm{a}}\end{array}\right)$

Herewith the CF current is given by:

$\boldsymbol{J}_{0}=\frac{1}{\mu_{0}}\left(\begin{array}{c}B_{0, \mathrm{a}} \cos \theta_{B}-B_{0, \mathrm{~b}} \\ -B_{0, \mathrm{a}} \sin \theta_{B}\end{array}\right)$

and $\delta \boldsymbol{J}$ can be written in the following form using the dispersion relation (18) and the boundary condition (12):

$$
\begin{aligned}
\delta \boldsymbol{J} & =\frac{2 \tilde{\tau}}{\mu_{0} \rho_{0, \mathrm{~b}} \Omega_{\mathrm{b}}^{2}} e^{i\left(k_{\mathrm{y}} y-\omega t\right)}\left\{\left(\begin{array}{c}
k_{\mathrm{y}} k_{\mathrm{z}} \cos \left(k_{\mathrm{z}} z\right) \\
-i k_{\mathrm{y}}^{2} \sin \left(k_{\mathrm{z}} z\right)
\end{array}\right) B_{0, \mathrm{a}} \sin \theta_{B}+\right. \\
& \left.+\left(\begin{array}{c}
i k_{\mathrm{z}}^{2} \sin \left(k_{\mathrm{z}} z\right) \\
-k_{\mathrm{y}} k_{\mathrm{z}} \cos \left(k_{\mathrm{z}} z\right)
\end{array}\right)\left[B_{0, \mathrm{a}} \cos \theta_{B}+B_{0, \mathrm{~b}}\right]\right\}
\end{aligned}
$$

This vector contains the $y$ - and $z$-components of the surface current density. The surface divergence of the current can be calculated therewith:

$$
\nabla \cdot \delta \boldsymbol{J}=\frac{\partial}{\partial y} \delta J_{\mathrm{y}}+\frac{\partial}{\partial z} \delta J_{\mathrm{z}}=0
$$

As expected from the current densities (44) inside of the two half spaces, the divergence of the surface current on the magnetopause vanishes.

\section{Validity of the calculations}

The purpose of this section is to discuss under which conditions the above given calculations are valid. First, a possible conflict between the dispersion relations of the magnetosonic waves and the KS-modes has to be considered in detail: The wave number of the reflected surface wave $(\mathrm{R})$ differs from the incident wave (I) to the ionosphere only by the sign of its z-component: $k_{\mathrm{z}}^{\mathrm{R}}=-k_{\mathrm{z}}^{\mathrm{I}}$. This is a direct consequence of the dispersion relation (17) in the incompressible case considered; assuming compressibility $k_{\mathrm{z}}^{\mathrm{R}}$ could be obtained from the more general relation (11) for magnetosonic waves. The frequency $\omega$ must be equal for both waves, which potentially 
conflicts with the dispersion relation (18) for the KS-modes, from which the frequency may be obtained for a given wave vector. If a common frequency holds for the two waves, either Eq. (17) or (18) may not be satisfied. Assuming the validity of Eq. (17) $k_{\mathrm{z}}^{\mathrm{R}}=-k_{\mathrm{z}}^{\mathrm{I}}$ the dispersion relation (18) is consistent herewith only, if $\left(k_{\mathrm{y}} \sin \theta_{B}+k_{\mathrm{z}} \cos \theta_{B}\right)^{2}$ is equal for both waves or $B_{0, \mathrm{a}}=0$ in the magnetosheath, which would be an unrealistic assumption. Since $k_{\mathrm{y}}$ has to remain unchanged for the boundary conditions at the ionospheres to be fulfilled, the above stated condition is, hence, only satisfied, if $k_{\mathrm{y}}=0$ (case 1$), \sin \theta_{B}=0$ (case 2 ) or $\cos \theta_{B}=0$ (case 3 ).

Additionally, it has to be tested if the assumption of an incompressible plasma is justified. For this purpose Eq. (11) may be used, which converts to Eq. (17) in the limit of incompressibility. The first term on the right side of Eq. (11):

$K^{2}:=\frac{\omega^{2}}{V_{\mathrm{A}}^{2}+C_{\mathrm{S}}^{2} \Omega^{2} / \omega^{2}}$

has to be much smaller than the second term on this side:

$\left|K^{2}\right| \ll k_{\mathrm{t}}^{2}$

It shall be noted, that $K$ can be negative (see definition of $\Omega^{2}$ ).

In order to make a realistic assessment of the relation (50) typical values of the plasma parameters $p_{B}$ (magnetic pressure), $N_{\mathrm{p}}$ (proton number density) and $\beta$ (quotient of thermal and magnetic pressures) on both sides of the magnetopause are used: We obtain these for the low shear (high shear) situation at the magnetopause from the Figs. 9 and 10 of Phan et al. (1994); they are listed in Table 1. Averages for the low and high shear situations are computed for each half space. From $p_{B}$ and $N_{\mathrm{p}}$ typical values of the background magnetic fields and of the mass densities can be calculated. For the magnetosheath we obtain: $B_{0, \mathrm{a}}=33.1 \mathrm{nT}$ and $\rho_{0, \mathrm{a}}=3.18 \times 10^{-20} \mathrm{~kg} \mathrm{~m}^{-3}$. It follows an Alfvén velocity of: $V_{\mathrm{A}, \mathrm{a}}=166 \mathrm{~km} \mathrm{~s}^{-1}$. The sound speed can then be computed with $\beta_{\mathrm{a}}=2.75: \quad C_{\mathrm{S}, \mathrm{a}}=V_{\mathrm{A}, \mathrm{a}} \sqrt{\gamma \beta_{\mathrm{a}} / 2}=251 \mathrm{~km} \mathrm{~s}^{-1}$. Here an adiabatic coefficient of $\gamma=5 / 3$ has been used. For the magnetosphere (half space " $\mathrm{b}$ ") we get the following values: $B_{0, \mathrm{~b}}=61.3 \mathrm{nT}, \rho_{0, \mathrm{~b}}=3.35 \times 10^{-21} \mathrm{~kg} \mathrm{~m}^{-3}, \beta_{\mathrm{b}}=0.15$, $V_{\mathrm{A}, \mathrm{b}}=945 \mathrm{~km} \mathrm{~s}^{-1}$ and $C_{\mathrm{S}, \mathrm{b}}=334 \mathrm{~km} \mathrm{~s}^{-1}$.

As can be easily seen, the relative importance of the sound speed $C_{\mathrm{S}}$ is greater in the magnetosheath, where it is larger than the Alfvén velocity. However in absolute terms both characteristic speeds (Alfvén and sound speed) are larger in the magnetosphere than in the magnetosheath. A typical length along the magnetopause between the ionospheres is taken from Plaschke et al. (2009b):

$z_{0}=2.73 \times 10^{5} \mathrm{~km}$

According to the relation for standing KS-modes (27), the field parallel wave number (on the magnetospheric side) is chosen to be $k_{\mathrm{z}}=n \pi / z_{0}$.

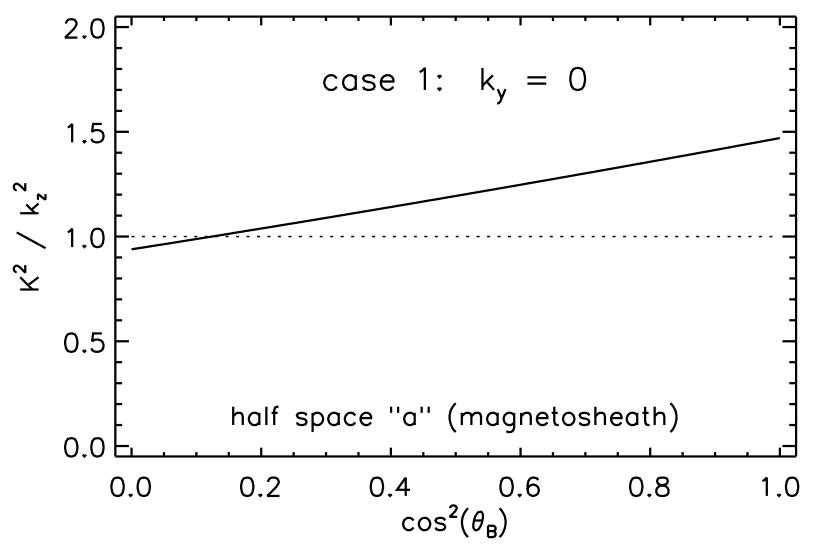

Fig. 2. Ratios $K^{2} / k_{\mathrm{z}}^{2}=K^{2} / k_{\mathrm{t}}^{2}$ of half space "a" (magnetosheath) for different values of $\cos ^{2} \theta_{B}$ and typical plasma conditions given in the text. Case $1\left(k_{\mathrm{y}}=0\right)$ has been considered. For low ratios $K^{2} / k_{\mathrm{z}}^{2} \ll 1$ the assumption of incompressibility would be justified.

We first consider case 1 and set $k_{\mathrm{y}}=0$ as well as the values given above in $K^{2}, k_{\mathrm{t}}^{2}$ and in the dispersion relation of the KS-modes (18). From this relation the corresponding eigenfrequencies $\omega$ of the standing KS-modes are obtained. Please note that in the cases 1 and 2, $\omega^{2}$ is proportional to $n^{2}$ and weakly dependent on $\cos ^{2} \theta_{B}$; this holds true also for $K^{2}$ (Eq. 49), which is a function of $\omega^{2}$. The ratio of $K^{2}\left(\cos ^{2} \theta_{B}\right)$ and $k_{\mathrm{t}}^{2}$ is shown in Fig. 2 for half space "a" (magnetosheath), where $k_{\mathrm{t}}^{2}=k_{\mathrm{z}}^{2}$ for $k_{\mathrm{y}}=0$. This quotient is no longer dependent on $n$; hence, it is valid for all the harmonics of the standing KS-modes under the above given assumptions (case 1) and parameters. The assumption of incompressibility would be justified, if the quotient were far below $1\left(K^{2} \ll k_{\mathrm{z}}^{2}\right)$. In Fig. 2, however, it can be seen that in half space "a" this quotient is mostly even larger for all the orientations of the magnetic field $\theta_{B}$ in the magnetosheath. Therefore, we have to conclude that the assumption of incompressibility is not valid for typical plasma conditions at the magnetopause if $k_{\mathrm{y}}=0$ is chosen.

Let us now consider case $3\left(\cos \theta_{B}=0\right)$, where $\sin \theta_{B}= \pm 1$ necessarily holds. The background magnetic field in the magnetosheath (half space "a") is, hence, assumed to be perpendicularly directed with respect to the field within the magnetosphere. As described, the quotient $K^{2} / k_{\mathrm{t}}^{2}$ can be computed with the above given plasma parameters. However, in this case the quotient is only dependent on $k_{\mathrm{y}}$ and no longer on $\theta_{B}$. If $k_{\mathrm{y}}$ is chosen as a factor of $k_{\mathrm{z}}$, which is proportional to $n$, it becomes itself proportional to the number of the harmonic. Thus, $K^{2}\left(k_{\mathrm{y}} / k_{\mathrm{z}}\right), \omega^{2}\left(k_{\mathrm{y}} / k_{\mathrm{z}}\right)$ and $k_{\mathrm{t}}^{2}\left(k_{\mathrm{y}} / k_{\mathrm{z}}\right)$ will be proportional to and the quotient $K^{2} / k_{\mathrm{t}}^{2}$ independent from $n^{2}$. This quotient is depicted in Fig. 3 for the magnetosheath. It can be seen that also in this case 3 the assumption of incompressibility $\left(K^{2} \ll k_{\mathrm{t}}^{2}\right)$ can hardly be justified, since ratios around 0.7 or larger are obtained for typical plasma conditions around the magnetopause. 
Table 1. Typical values of the magnetic pressure $p_{B}$, the proton number density $N_{\mathrm{p}}$ and the quotient of thermal and magnetic pressures $\beta$ on both sides of the magnetopause for the low and high shear situations. Taken from the Figs. 9 and 10 of Phan et al. (1994).

\begin{tabular}{lccccc}
\hline & \multicolumn{2}{c}{ low shear } & & \multicolumn{2}{c}{ high shear } \\
\cline { 2 - 3 } \cline { 5 - 6 } quantity & $\begin{array}{c}\text { magnetosheath } \\
\text { (half space "a") }\end{array}$ & $\begin{array}{c}\text { magnetosphere } \\
\text { (half space "b") }\end{array}$ & & $\begin{array}{c}\text { magnetosheath } \\
\text { (half space "a") }\end{array}$ & $\begin{array}{c}\text { magnetosphere } \\
\text { (half space "b") }\end{array}$ \\
\hline$p_{B}$ & $0.6 \mathrm{nPa}$ & $1.7 \mathrm{nPa}$ & & $0.3 \mathrm{nPa}$ & $1.3 \mathrm{nPa}$ \\
$N_{\mathrm{p}}$ & $20 \mathrm{~cm}^{-3}$ & $3 \mathrm{~cm}^{-3}$ & & $18 \mathrm{~cm}^{-3}$ & $1 \mathrm{~cm}^{-3}$ \\
$\beta$ & 2.0 & 0.2 & & 3.5 & 0.1 \\
\hline
\end{tabular}

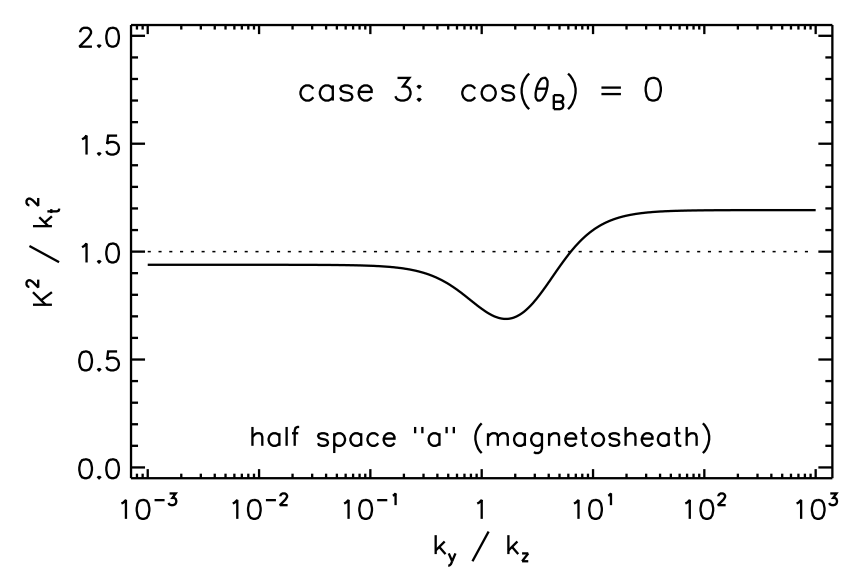

Fig. 3. Ratios $K^{2} / k_{\mathrm{t}}^{2}=K^{2} /\left(k_{\mathrm{y}}^{2}+k_{\mathrm{z}}^{2}\right)$ of half space "a" (magnetosheath) for different values of $k_{\mathrm{y}} / k_{\mathrm{z}}$ and typical plasma conditions given in the text. Case $3\left(\cos \theta_{B}=0\right)$ has been considered. For low ratios $K^{2} / k_{\mathrm{t}}^{2} \ll 1$ the assumption of incompressibility would be justified.

The only remaining option for all equations to be strictly satisfied is case $2\left(\sin \theta_{B}=0\right)$ : In this case both frequencies $\omega^{2}$ and $\Omega^{2}$ are not dependent on $k_{\mathrm{y}}$; hence, this holds true also for $K^{2}$. If $k_{\mathrm{y}}$ is increased, $k_{\mathrm{t}}^{2}=k_{\mathrm{y}}^{2}+k_{\mathrm{z}}^{2}$ will grow and the ratio $\left|K^{2}\right| / k_{\mathrm{t}}^{2}$ will decrease correspondingly such that the relation (50) can be satisfied in both half spaces. Therefore, if $\sin \theta_{B}=0$ yields (case 2 ), a sufficiently large $k_{\mathrm{y}}$ will result in the incompressibility assumption to be globally justified.

The results of the preceding section are, hence, only applicable, if the magnetic field in the magnetosheath is parallel or antiparallel to the magnetospheric background field. As already stated, the dispersion relation of the surface KSmodes (Eq. 18) becomes then independent from $k_{\mathrm{y}}$; the eigenfrequencies of these modes remain only dependent on the plasma parameters in both half spaces and the distance between the ionospheres $z_{0}$ along the magnetopause field lines.

The validity of the calculations is further limited due to the simplified geometry of the box magnetosphere and also due to the assumption of a vanishing background velocity in both half spaces $\left(\boldsymbol{v}_{0}=0\right)$. It shall be recalled that the plasma flow velocity increases in the actual magnetosheath with increasing distance from the stagnation point towards the tail. If the plasma convection inside the inner magnetosphere is neglected, a fairly good approximation to this situation could be achieved in the box magnetosphere model, if a radially outward directed magnetosheath plasma flow tangential to the magnetopause would be assumed diverging from a central (stagnation) point at $z=z_{0} / 2$.

The theory of the KS-modes can be generalized to include effects of a constant shear flow across the boundary as shown for instance in $\mathrm{Pu}$ and Kivelson (1983) by taking into account a doppler shift of the frequency in one of the two half spaces; if only a flow in the magnetosheath half space "a" is present, this relation would be:

$\omega_{\mathrm{a}}=\omega_{\mathrm{b}}-\boldsymbol{k}_{\mathrm{t}} \cdot \boldsymbol{v}_{0, \mathrm{a}}$

Here $\omega_{\mathrm{a}}$ and $\omega_{\mathrm{b}}$ are the frequencies in the respective plasma rest frames and $\boldsymbol{v}_{0, \mathrm{a}}$ is the background flow velocity vector (tangential to the magnetopause) in the magnetosheath. By substitution of $\omega_{\mathrm{a}}$ with Eq. (52) in $\Omega_{\mathrm{a}}^{2}$ of Eq. (18) a modified dispersion relation for the KS-modes can be obtained (see Eq. 53 below) as computed by Pu and Kivelson (1983).

Although the propagation of the KS-modes is in principle possible in the presence of a magnetosheath plasma flow, finding a solution of the MHD equations for the complex (divergent) flow situation suggested above would most probably have to be subject to numerical calculations and, thus, out of the scope of this paper. Therefore, we opted for considering only the case of a vanishing background plasma velocity, which describes best the situation close to the stagnation point on the terrestrial magnetopause.

Additionally, a strong shear flow would be quite unfavorable for the appearance of standing KS-modes: first, the boundary could become Kelvin-Helmholtz unstable. In this case the perturbations caused by a propagating KS-mode could grow in amplitude fed by the shear flow; the wave forms would at some point be distorted due to non-linear effects diminishing the constructive self-interference of the $\mathrm{KS}$-mode after reflection at the ionospheres, which is required for a standing wave to occur.

Secondly, the larger the flow is on the magnetosheath side the more difficult it should be for a KS-mode to propagate 
in opposite direction to this flow. This shall be considered in detail: In case 2 (parallel magnetic fields in both half spaces) the dispersion relation (18) of the KS-modes for a non-vanishing flow in the z-direction $\left(\boldsymbol{v}_{0, \mathrm{a}}=v_{0, \mathrm{a}} \boldsymbol{e}_{\mathrm{Z}}\right)$ is given by:

$$
\begin{aligned}
\frac{\omega_{\mathrm{b}}^{2}}{k_{\mathrm{z}}^{2}}\left(\rho_{0, \mathrm{a}}+\rho_{0, \mathrm{~b}}\right) & -\frac{\omega_{\mathrm{b}}}{k_{\mathrm{z}}} 2 \rho_{0, \mathrm{a}} v_{0, \mathrm{a}}+ \\
& +\rho_{0, \mathrm{a}} v_{0, \mathrm{a}}^{2}-\rho_{0, \mathrm{a}} V_{\mathrm{A}, \mathrm{a}}^{2}-\rho_{0, \mathrm{~b}} V_{\mathrm{A}, \mathrm{b}}^{2}=0
\end{aligned}
$$

This flow situation may be apparent at the real terrestrial magnetopause north or south of the stagnation point towards the cusp regions. The solutions in $\omega_{\mathrm{b}} / k_{\mathrm{z}}$ of this quadratic equation correspond to the propagation speeds parallel or antiparallel to the flow in the z-direction in the frame of the resting magnetospheric plasma. They are depicted in Fig. 4 by solid lines as a function of $v_{0, \mathrm{a}}$ for the typical values of $\rho_{0, \mathrm{a}}$, $\rho_{0, \mathrm{~b}}, V_{\mathrm{A}, \mathrm{a}}$ and $V_{\mathrm{A}, \mathrm{b}}$ derived from Phan et al. (1994). The + and - signs indicate the flow-parallel and anti-parallel propagation speeds. For comparison the function $\omega_{\mathrm{b}} / k_{\mathrm{z}}=v_{0, \mathrm{a}}$ is shown with a dashed line; $\omega_{\mathrm{b}} / k_{\mathrm{z}}=0$ is depicted by a dotted line.

As can be seen, the flow increases the propagation speed of the KS-modes in its direction whereas oppositely propagating KS-modes move slower than without a magnetosheath flow. In this case of vanishing flow velocity $\left(v_{0, a}=0\right)$ the propagation is symmetric in the z-direction at (see Plaschke et al., 2009b):

$v_{\mathrm{ph}}:=\omega_{\mathrm{b}} / k_{\mathrm{z}}=332 \mathrm{~km} \mathrm{~s}^{-1}$

given by the typical magnetosheath and magnetospheric plasma parameters indicated above. If the flow speed reaches a critical value (here approximately $v_{0, \mathrm{a}}=350 \mathrm{~km} \mathrm{~s}^{-1}$ ) then KS-modes are no longer able to propagate against the flow in the magnetospheric plasma rest frame and standing KSmodes cannot occur. This shows that the concept of the standing KS-modes is not applicable any more if at any point along the magnetopause any of the two oppositely propagating waves composing the standing KS-mode is not able to propagate against the magnetosheath plasma flow as its speed may exceed the critical value. Clearly, a slow solar wind leading to diminished plasma flow velocities in the magnetosheath appears to be favorable for standing KS-modes to occur, as suggested also by the results of Plaschke et al. (2009c).

\section{Discussion and properties of the KS-modes}

The ansatz used in Sect. 3 for the standing KS-modes, where their reflection at infinitely conducting ionospheric layers is assumed, is not new. It has been used for instance for the computation of standing Alfvén waves in the magnetosphere (e.g. Cummings et al., 1969). In a box magnetosphere model of the situation the disturbance in the electric field and the

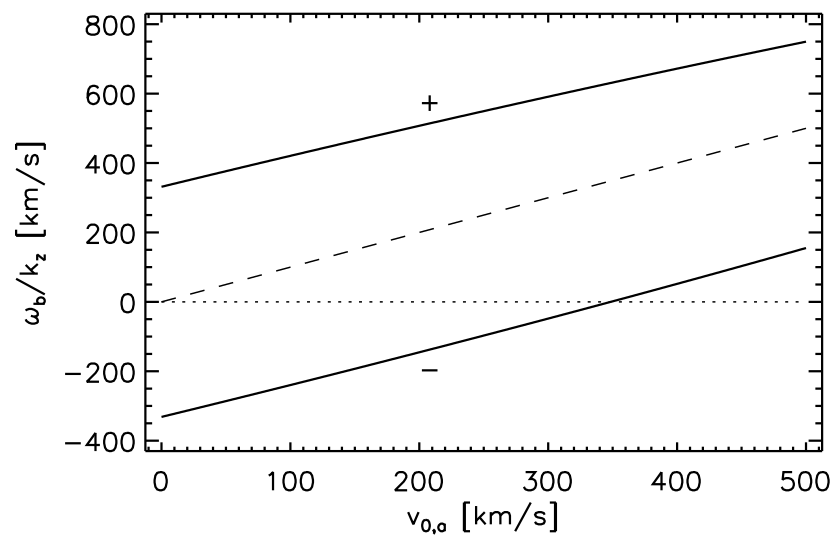

Fig. 4. Solid lines: propagation speeds of a KS-mode in the $\mathrm{z}$ direction $\left(\omega_{\mathrm{b}} / k_{\mathrm{Z}}\right)$ in the frame of the resting magnetospheric plasma as a function of the magnetosheath flow speed $v_{0, \text { a }}$ (also in the $\mathrm{z}$ direction). \pm : parallel or anti-parallel propagation of the KS-mode with respect to the flow. Dashed line: function $\omega_{\mathrm{b}} / k_{\mathrm{z}}=v_{0, \mathrm{a}}$, dotted line: $\omega_{\mathrm{b}} / k_{\mathrm{z}}=0$.

velocity for a standing Alfvén wave would be proportional to a factor $\sin \left(k_{\mathrm{z}} z\right)$, which maximizes in the equatorial magnetosphere in the case of the fundamental mode. The amplitude of the magnetic field oscillations, however, would be strongest in the vicinity of the ionospheres; their amplitude would be proportional to a factor $\cos \left(k_{\mathrm{z}} z\right)$.

In the case of the KS-modes the amplitude distributions are not as simple: The reflection takes place only on one side of the magnetopause, while the plasma parameters on both sides are important for the propagation of the KS-modes. The sine and cosine factors are both part of the amplitudes of the velocity and magnetic field disturbances, depending on the component, and are both present in each component of $\delta \boldsymbol{B}_{\mathrm{a}}$ and $\delta \boldsymbol{J}$, which makes the situation more complex. These differences simply arise from the fact that KS-modes are not plain Alfvén waves: The governing dispersion relation in both half spaces belongs to the magnetosonic waves. Nevertheless, the standing KS-mode does have some remarkable similarities with a standing Alfvén wave, which will be discussed in this section.

We begin with an interpretation of $\delta \boldsymbol{B}_{\mathrm{a}}$ and $\delta \boldsymbol{B}_{\mathrm{b}}$ (Eqs. 43 and 35 ). According to the results of the previous section the background magnetic field in the magnetosheath (half space "a") is assumed to be parallel to the magnetospheric field: $\boldsymbol{B}_{0, \mathrm{a}}=B_{0, \mathrm{a}} \boldsymbol{e}_{\mathrm{z}}$. It follows that $\theta_{B}=0, \sin \theta_{B}=0$ and $\cos \theta_{B}=$ 1. This simplifies considerably the relations for $\delta \boldsymbol{B}_{\mathrm{a}}$ (Eq. 43) and $\delta \boldsymbol{J}$ (Eq. 47). Furthermore, only the fundamental mode of the standing KS-modes shall be discussed $(n=1)$. From Eq. (27) we obtain: $k_{\mathrm{z}} z_{0}=\pi$.

Under these assumptions the field perpendicular components of the disturbances of the magnetic field ( $\mathrm{x}-$ and $\mathrm{y}$ components of $\delta \boldsymbol{B}_{\mathrm{a}}$ and $\delta \boldsymbol{B}_{\mathrm{b}}$ ) are strong only in the vicinity of the ionospheres. In Eq. (35) this is apparent in the 


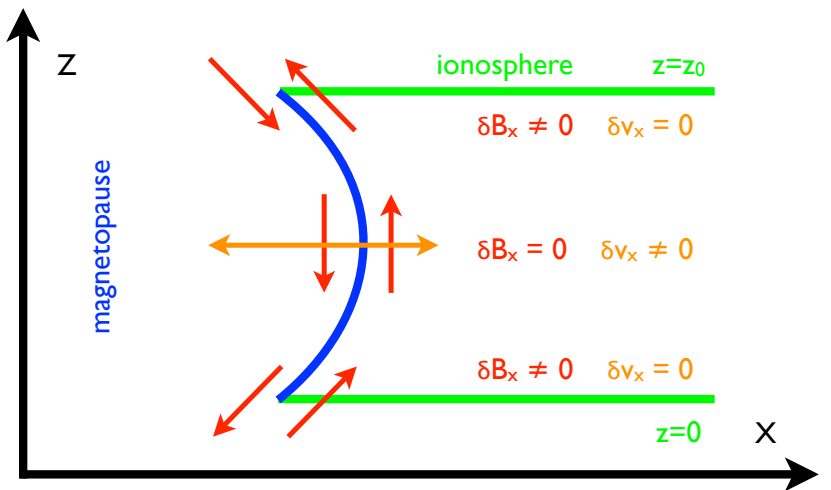

Fig. 5. Sketch of the magnetic and velocity fields and of the magnetopause inclination. In the equatorial plane the radial velocity of the magnetopause $\left(\delta v_{\mathrm{X}}\right)$ is strongest. At the ionospheres no boundary motion takes place. The consequent inclination of the magnetopause corresponds with the inclination of the magnetic field in both half spaces, such that it remains tangential to the boundary.

factor $\cos \left(k_{\mathrm{z}} z\right)$ of these components, which maximizes at $z=0$ and $z=z_{0}$. This applies also to Eq. (43), where due to $\sin \theta_{B}=0$ only the second terms of the sums within the vector are relevant. However, the field parallel components (zdirection) in both half spaces vanish at $z=0$ and $z=z_{0}$ due to the factor $\sin \left(k_{\mathrm{z}} z\right)$. Hence, the disturbances of the magnetic field close to the ionospheres are purely field perpendicular and the standing KS-modes look almost like standing Alfvén waves there. In the vicinity of the equatorial plane the character of the KS-mode is completely different: The amplitudes of the z-components (field-parallel) of the disturbances of the magnetic field maximize on both sides of the magnetopause; the field perpendicular components vanish. Hence, the oscillations in the magnetic fields are purely compressional there. This difference in the character of the magnetic field disturbances depending on the location (Alfvénic at the ionospheres, compressional at the equator) is one of the most remarkable properties of the standing KS-modes.

The oscillations in the velocity fields correspond with the results obtained for the magnetic fields via the induction Eq. (3). Unlike for $\delta \boldsymbol{B}_{\mathrm{a}}$ the direction of the background magnetic field in the magnetosheath $\left(\theta_{B}\right)$ is generally irrelevant for $\delta \boldsymbol{v}$. As already known for standing Alfvén waves in the magnetosphere, the amplitudes of $\delta \boldsymbol{v}$ maximize where the ones of $\delta \boldsymbol{B}$ do not and vice versa. Accordingly, the field-perpendicular components are strongest in the equatorial plane (sine factor); the z-component (field parallel) maximizes at the ionospheres.

As computed above the standing KS-mode is associated with a shear tangential plasma flow across the magnetopause boundary (see Eq. 39). Near the ionospheres only a field parallel plasma motion (z-component) takes place in the fundamental KS-mode. In the equatorial plane, however, the azimuthal y-component of this shear flow is strongest. Fur- thermore, it is potentially Kelvin-Helmholtz unstable under the assumption of parallel background magnetic fields in the z-direction. The implications this may have to the KS-mode are unclear at this point; further studies are required to determine possible adverse effects on the stability of the KS-mode or any changes in its properties if the Kelvin-Helmholtz instability is taken into consideration.

The continuity of the $\mathrm{x}$-component of the plasma velocity has been set as boundary condition; $\delta v_{\mathrm{x}}$ (Eqs. 32 and 40) also represents the radial velocity of the magnetopause boundary itself. Its amplitude distribution is consistent with the expectation, that the most notable motion of the magnetopause should occur in the equatorial plane; the boundary is fixed by the magnetospheric magnetic field at the ionospheres and, hence, its motion shall be minimal there, as depicted in Fig. 5. The inhomogeneous radial velocity field leads to an inclination of the boundary, which is largest near the ionospheres in the geometry of the box magnetosphere. In the real magnetosphere the points of negligible magnetopause (radial) motion would correspond to the northern and southern cusp regions. At the magnetic equator, however, where the magnetopause moves in normal direction, no inclination in the x-z-plane is observed for the fundamental KS-mode.

Interestingly, although the $\delta v_{\mathrm{x}}$ component is continuous across the magnetopause, $\delta B_{\mathrm{x}}$ is not. This raises the question if the condition $\nabla \cdot \boldsymbol{B}=0$ may not be satisfied at the boundary. With the dispersion relation (16) and under the assumption of parallel background magnetic fields $\left(\theta_{B}=0\right)$ we obtain from the $\mathrm{x}$-components of the Eqs. (35) and (43) at the position of the magnetopause $(x=0)$ :

$\delta B_{\mathrm{x}, \mathrm{b}} / B_{0, \mathrm{~b}}=\delta B_{\mathrm{x}, \mathrm{a}} / B_{0, \mathrm{a}}$

This result is consistent with the radial velocity $\delta v_{\mathrm{x}}$ and the inclination of the magnetopause discussed above (see Fig. 5). The amplitudes of the $\delta B_{\mathrm{x}}$ components are largest at the ionospheres, where the inclination of the magnetopause is largest. Since the quotient of the disturbance in that direction and the background magnetic field (in z-direction as assumed) is continuous across the magnetopause, the total magnetic field vectors in the X-Z-plane at the boundary remain tangential to each other on both sides and consistent with the magnetopause inclination (see Fig. 6). This can be easily proven: The displacement $\xi_{\mathrm{x}}$ of the boundary (in $\mathrm{x}$-direction) can be obtained from the velocity $v_{\mathrm{x}}$ (e.g. De Keyser et al., 1999):

$-i \omega \xi_{\mathrm{x}}=v_{\mathrm{x}}$

It is a function of the coordinates $y$ and $z: \xi_{\mathrm{x}}=\xi_{\mathrm{x}}(y, z)$. A vector normal to the boundary $\boldsymbol{n}$ is given at any point $(y, z)$ by:

$\boldsymbol{n}=\left(\begin{array}{c}1 \\ -\partial \xi_{\mathrm{x}} / \partial y \\ -\partial \xi_{\mathrm{x}} / \partial z\end{array}\right)$ 


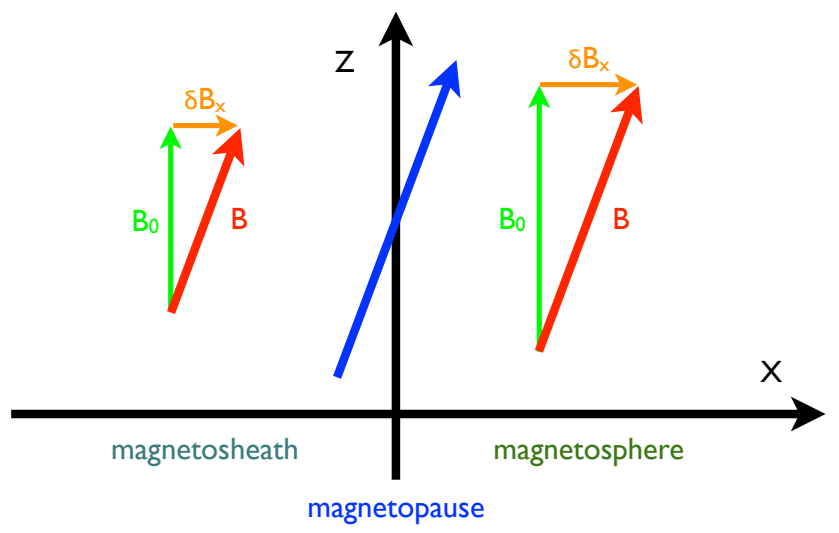

Fig. 6. Sketch of the magnetic field in the $x-z-$ plane: The total field is tangential to the magnetopause on both sides, such that the normal component vanishes. The magnetopause keeps being a tangential discontinuity. The inclination of the magnetic fields corresponds with the local inclination of the magnetopause.

For the total magnetic field to be parallel to be magnetopause, it must obey the relation $\boldsymbol{n} \cdot \boldsymbol{B}=0$. This gives with $B_{0 \mathrm{x}}=0$ independently of the half space considered:

$$
\begin{aligned}
0 & =\boldsymbol{n} \cdot \boldsymbol{B}_{0}+\boldsymbol{n} \cdot \delta \boldsymbol{B} \\
& =-\left(\boldsymbol{B}_{0} \cdot \nabla\right) \xi_{\mathrm{x}}+\delta B_{\mathrm{x}}-\frac{\partial \xi_{\mathrm{x}}}{\partial y} \delta B_{\mathrm{y}}-\frac{\partial \xi_{\mathrm{x}}}{\partial z} \delta B_{\mathrm{z}}
\end{aligned}
$$

Linearization of this Eq. (59) yields the omission of its last two terms, because they consist of a product of two perturbed quantities. Thus we obtain:

$\left(\boldsymbol{B}_{0} \cdot \nabla\right) \xi_{\mathrm{x}}=\delta B_{\mathrm{x}}$

Replacing $\xi_{\mathrm{x}}$ with $v_{\mathrm{x}}$ via Eq. (56) yields exactly the $\mathrm{x}$ component of the induction Eqs. (33) and (41) for the half spaces "b" and "a". Hence, in the framework of linearized MHD theory the magnetopause strictly keeps being a tangential discontinuity without any magnetic field component normal to its surface, where $\nabla \cdot \boldsymbol{B}=0$ is satisfied.

We now focus on the disturbance of the surface current density: if $\sin \theta_{B}=0$ is assumed, the background surface current $\boldsymbol{J}_{0}$ flows everywhere on the magnetopause surface in y-direction (CF current). The disturbance $\delta \boldsymbol{J}$ is given by Eq. (47), of which only the second term in the brackets (proportional to $B_{0, \mathrm{a}} \cos \theta_{B}+B_{0, \mathrm{~b}}$ ) is important. Interestingly, if $\cos \theta_{B}=1$ and $B_{0, \mathrm{a}}=-B_{0, \mathrm{~b}}$ then $\delta \boldsymbol{J}=0$ follows (antiparallel background fields of equal strength). Otherwise, the $\mathrm{y}$-component of $\delta \boldsymbol{J}$ is strongest in the equatorial plane, where oscillations in its field parallel z-component vanish. Hence, the $\mathrm{CF}$ current is periodically increased and decreased in this plane, but not deflected. This corresponds to the oscillations in the z-component of the magnetic fields on both sides of the magnetopause.

At the boundary $(x=0)$ the $y$ - and z-components of $\delta \boldsymbol{B}$ (Eqs. 35 and 43 ) obey the following relation assuming $\theta_{B}=0$ :

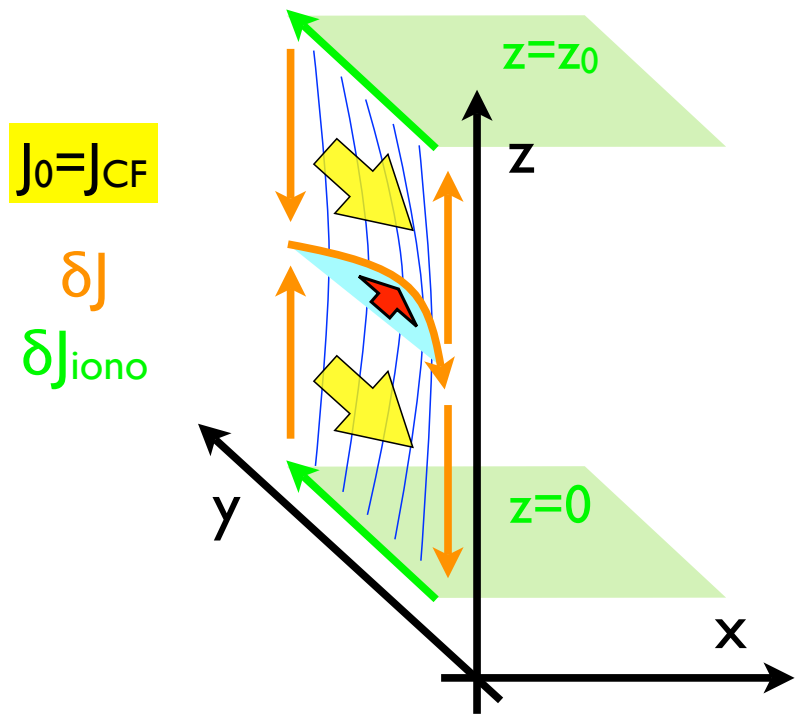

Fig. 7. Current system in the fundamental KS-mode. The indentation of the equatorial magnetopause (red arrow) is associated with a disturbance surface current (orange arrows), which increases the $\mathrm{CF}$ current (yellow arrows). The localization of the indentation makes the currents to be closed via field aligned currents to the ionosphere, where they are closed via ionospheric currents (green arrows).

$\frac{\delta B_{\mathrm{y} / \mathrm{z}, \mathrm{a}}}{B_{0, \mathrm{a}}}=-\frac{\delta B_{\mathrm{y} / \mathrm{z}, \mathrm{b}}}{B_{0, \mathrm{~b}}}$

When the magnetopause moves in the equatorial plane, the magnetic field will be compressed on one side and expanded on the other. Hence, if $B_{0, \mathrm{a}}$ and $B_{0, \mathrm{~b}}$ are both positive (parallel background fields), $\delta B_{\mathrm{Z}}$ must have opposite signs on both sides consistent with Eq. (61). If the background fields are anti-parallel, the expansion and compression correspond to magnetic field disturbances $\delta B_{\mathrm{Z}}$ in equal direction: the negative sign in Eq. (61) is countered by the opposite signs of the background fields $B_{0}$.

In the vicinity of the ionospheres only the field parallel zcomponent of $\delta \boldsymbol{J}$ remains: The superposed, additional current to the CF current in the equatorial plane has to be closed somehow. Since the surface divergence has been found to be zero (Eq. 48) and also the current densities vanish in both half spaces (Eq. 44), this closure can only be achieved via field aligned currents in the magnetopause from and towards the ionospheres at $z=0$ and $z=z_{0}$. In this aspect the standing KS-modes are similar to standing Alfvén waves, whose field aligned currents also have to be closed in the ionosphere. The field aligned currents themselves are associated with the Alfvénic magnetic disturbances near the ionospheres, which have already been discussed. The complete current circuit on the magnetopause and in the ionospheres corresponding to an inward motion of the boundary is depicted in Fig. 7.

As already mentioned, the current density vanishes within the half spaces (Eq. 44). Therein only evanescent waves propagating along $z$ in opposite directions can be observed, 
which belong together to the standing KS-mode structure described. The KS-mode is, hence, a pure surface wave, which is supported by currents flowing entirely inside of the boundary (closed via the ionospheres); any divergence of these surface currents would lead to mode coupling of the KS-mode to propagating waves inside of any of the two half spaces. The motion of the magnetopause associated with a KS-mode is very similar to that of a membrane under tension: The KS-modes correspond in this picture to the membrane eigenmodes. The wave number $k_{\mathrm{z}}$ along the background field accounts for the quantization of these eigenmodes. The standing KS-modes are possible due to their reflection at the ionospheres, where the magnetopause is at rest and the oscillating (magnetopause) membrane is supported.

The current system and the characteristics of these membrane eigenoscillations match the excitation mechanism of the KS-modes described in Plaschke et al. (2009b): A local indentation of the magnetopause leads to a local disturbance of the CF current, which is closed via field aligned currents associated with an Alfvénic disturbance of the magnetic field (Glassmeier and Heppner, 1992). The localization of the indentation is emphasized here, because a global motion of the magnetopause would only result in a global modification of the strength of the CF current without a qualitative modification of the current configuration. The field aligned currents are closed in the ionosphere, if they flow on closed magnetopause field lines. Hence, the current system set up by a local indentation of the magnetopause matches the one associated with the KS-mode as described above (see Fig. 7). Consequently, a localized pressure enhancement in the vicinity of the dayside magnetopause within the magnetosheath should be favourable for the excitation of a standing KS-mode.

This mechanism of excitation is in some respects similar, however, ultimately different to the field-line resonance (FLR) process, by which standing Alfvén waves may be generated inside the magnetosphere; the FLR process shall be briefly recalled (see Southwood, 1974): if compressibility of the plasma is assumed, then compressional waves traveling from the magnetopause in the direction of the Alfvén velocity gradient into the magnetosphere (towards the Earth) may encounter a turning point where they are totally reflected. Beyond this point the character of the compressional waves changes from propagating to evanescent. Nevertheless, further inside the evanescent compressional waves can couple locally to Alfvén waves at a resonance point, where their propagation velocity in the direction of the magnetic field equals the Alfvén velocity (resonant coupling, first resonance condition). Alfvén waves standing between the ionospheres may occur where their eigenfrequency coincides with the frequency of the driving compressional wave (second resonance condition). Since there is a smooth gradient in eigenfrequencies across field-line shells in the magnetosphere disturbances of a broad range of ultra-low frequencies may lead to the generation of standing Alfvén waves via the FLR process.
This process can also occur inside the magnetopause, if the boundary is considered to be of (small but) nonnegligible thickness, wherein a smooth transition between magnetosheath and magnetospheric values of all relevant plasma parameters takes place. In this case fast waves incoming from the magnetosheath may be reflected at turning points or couple locally to Alfvén or slow waves at (multiple) resonance points depending on the characteristics of the fast wave (the boundary tangential wave vector), the gradient of the Alfvén velocity and the rotation of the magnetic field inside the magnetopause. Different classes of possible solutions are discussed in detail in Belmont et al. (1995) and De Keyser et al. (1999).

The FLR and the excitation mechanism of KS-modes are similar with respect to the local coupling of a compressible disturbance (a fast wave or a pressure enhancement) to another mode (an Alfvén wave or a KS-mode), which absorbs part of the energy of the original disturbance and may evolve into a standing mode due to ionospheric reflection. One of the major differences are the modes taking part in the respective coupling processes: As stated above, the KS-mode is a collective mode of oscillation involving plasma on both sides of the boundary. It is composed of two evanescent magnetosonic waves, one in either of the two half spaces. The Alfvén wave generated by a FLR, instead, may propagate inside the magnetosphere or inside the magnetopause boundary only. Both waves (Alfvén and KS-modes) may be simultaneously present at the magnetopause. The Alfvén wave is generated via a FLR in a boundary of finite thickness, where the plasma parameters change smoothly from the magnetosheath to the magnetospheric side. The KS-mode, instead, will be exponentially damped unless the boundary has a vanishing thickness. The damping rate increases if the change in the plasma parameters is more gradual (less sharp), as Chen and Hasegawa (1974b) have shown.

The nature of the KS-mode actually opposes its excitation by a fast wave incoming from the magnetosheath: on this side of the boundary the KS-mode resembles an evanescent magnetosonic wave. A propagating fast wave would inevitably have to have the same tangential wave vector $\left(\boldsymbol{k}_{\mathrm{t}}\right)$ as the (evanescent) KS-mode to which it couples; the wave number $k_{\mathrm{x}}^{2}$ would also have to be equal for both waves (see Eq. 11). This, however, is impossible, since a wave cannot be at the same time evanescent (imaginary $k_{\mathrm{x}}$, amplitude decaying exponentially with distance from the magnetopause) and propagating (real $k_{\mathrm{x}}$ ). Thus, a KS-mode cannot be excited with a running fast wave via resonant coupling (FLR) inside of the boundary.

Due to the same reason a KS-mode cannot radiate its energy away by coupling to fast waves. In the real magnetopause a KS-mode will, however, be damped due to coupling to Alfvén waves inside the magnetopause boundary, as described above, due to ionospheric dissipation (Joule heating) or coupling to Alfvén waves inside the magnetosphere or magnetosheath regions in the presence of an Alfvén velocity 
gradient (non-uniform plasma conditions) or curvature of the magnetic field (Chen and Hasegawa, 1974a).

Another major difference between standing Alfvén waves generated via a FLR and standing KS-modes excited by local pressure enhancements in the magnetosheath is their range of possible frequencies: in the magnetosphere a smooth gradient in the Alfvén velocity directed towards Earth and the gradual change in field line length yields a smooth gradient in eigenfrequencies of Alfvén waves standing between the northern and southern ionospheres. This also holds for FLRs occurring inside the magnetopause due to the gradual change in the plasma parameters. The propagation speed of KS-modes, however, is determined by the plasma conditions on both sides of the boundary; if the geometry of the magnetopause is given (assumption of a fixed distance between the ionospheres along closed magnetopause field lines) there is in principle no range of eigenfrequencies KS-modes can be excited with. Instead there is a set of discrete eigenfrequencies for standing KS-modes. A calculation of those has already been presented in Plaschke et al. (2009b) for case 2 (parallel or antiparallel magnetic fields in both half spaces):

Assuming a fixed propagation speed along the magnetic field of $v_{\mathrm{ph}}=332 \mathrm{~km} \mathrm{~s}^{-1}$ (Eq. 54) and a distance between the ionospheres of $z_{0}=2.73 \times 10^{5} \mathrm{~km}$ (Eq. 51) a fundamental frequency of (Eq. 5 in Plaschke et al., 2009b):

$f=\frac{v_{\mathrm{ph}}}{2 z_{0}}=0.6 \mathrm{mHz}$

is obtained. It shall be noted that this is only an approximation to the real fundamental eigenfrequency of standing KSmodes; a slightly higher frequency of $0.65 \mathrm{mHz}$, for instance, would be in excellent agreement with the "magic" frequencies previously observed (harmonics at: 1.3, 1.95, 2.6 and $3.25 \mathrm{mHz}$ ). It is this coincidence of the frequencies which makes the standing KS-modes a promising candidate for the explanation of the prominent appearance of the "magic" frequencies in magnetospheric and ground measurements motivating the study of these modes.

\section{Summary and conclusions}

In this paper the structure of standing KS-modes (surface waves) on the magnetopause has been computed in the approximation of the ideal, single-fluid MHD for incompressible plasmas. The KS-modes consist of evanescent magnetosonic waves on both sides of the boundary; they are pure surface waves, as the currents supporting these waves flow entirely within the magnetopause. Standing KS-modes can occur, if they are reflected at both northern and southern ionospheres. This is particularly remarkable, as only the closed magnetospheric field lines on the inner side of the magnetopause are connected to the ionospheres. Consequently, the reflection of the KS-modes or, more precisely, the inner magnetospheric surface waves occurs only within the magnetosphere. However, the outer magnetosheath plasma is not disconnected from the magnetospheric side due to the magnetopause boundary conditions: the spatial structure of the magnetosheath waves is imposed by their magnetospheric counterparts, whose spatial structure is, in turn, determined by the ionospheric reflection. As a result, this reflection holds for the entire KS-mode and not only for the waves on one of the two sides. If reflection takes place at the two opposing ionospheres KS-modes can be trapped in between and a standing KS-mode can be established.

One of the restrictions for a standing KS-mode to occur is the consistency between the Eq. (11), the dispersion relation for magnetosonic waves, and (16), the dispersion relation for the KS-modes. These equations correspond with (17) and (18), if incompressibility of the plasma is assumed; they have been found to yield consistent results regarding $\boldsymbol{k}$ and $\omega$ for KS-modes reflected at the ionospheres under realistic magnetopause plasma conditions if the background magnetic fields adjacent to the dayside magnetopause are parallel or anti-parallel to each other.

Furthermore, the background plasma flow velocity has been assumed to vanish; on the magnetosheath side this is only strictly fulfilled along the stagnation streamline. As shown, a magnetosheath flow would enhance the propagation of KS-modes in the same direction, whereas the propagation speed in the opposite direction would be diminished. Hence, there is a critical plasma flow velocity beyond which KSmodes can only follow the flow, which possibly impedes the occurrence of standing KS-modes. An increased flow may also cause the boundary to become Kelvin-Helmholtz unstable which has been found to be adverse for the development of standing KS-modes.

In addition, damping of the KS-modes is not considered in the presented calculations: the ionosphere is treated as a perfectly reflecting boundary neglecting its Joule heating and the consequent dissipation of energy of the reflected KS-mode. The assumptions of a vanishing magnetopause boundary thickness and of homogeneous background plasma conditions on both sides thereof prevent any possible coupling between the standing KS-modes considered and Alfvén waves in the magnetosheath, the magnetosphere or inside of the magnetopause. Being this the case, energy could be transferred to Alfvén waves causing the amplitude of the KSmodes to decay with time.

Given the stated limitations, the structure of the KS-modes has been discussed assuming parallel background magnetic fields in the magnetosphere and the magnetosheath. These are our main findings:

In the fundamental mode the radial motion of the magnetopause takes place at the magnetic equator, where the disturbances in the magnetic fields are compressional and the magnetopause shows only minimal inclination. This inclination is most pronounced at the ionospheres, where the boundary is fixed. In contrast to the equatorial situation, the disturbances of the magnetic fields are purely field perpendicular 
there, resembling those of a standing Alfvén wave. The ionospheric footpoints of the closed magnetopause field lines can be identified with the supporting points of a magnetopause membrane, whose eigenmodes of oscillation correspond with the KS-modes discussed. Consequently, the magnetopause has been found to resemble a membrane under tension when moving in accordance with a standing KS-mode.

Remarkably, the inclinations of the total magnetic fields and the magnetopause agree at every location of the boundary, such that it remains a tangential discontinuity at all times. The radial motion of the magnetopause corresponds with a disturbance of the CF current. If this motion is localized, the additional current on the magnetopause may be closed via field aligned and ionospheric currents yielding the current system of a standing KS-mode. Hence, a localized pressure pulse generating a local indentation of the magnetopause can lead to the excitation of a standing KS-mode by setting up its current system.

Unlike for standing Alfvén waves in the magnetosphere there is no eigenfrequency gradient for standing KS-modes. Given the plasma conditions on both sides of the magnetopause and a fixed distance between the ionospheres along the boundary there is only one fundamental frequency. Multiples of this frequency (harmonics) computed for typical plasma parameters are found to be quite close to the so-called "magic" frequencies, such that the standing KS-modes can be regarded as a possible explanation for their prominent appearance in space and ground-based measurements of geomagnetic pulsations.

Apart from the theoretical considerations presented, the interesting question remains if these KS-modes are actually present at the Earth's magnetopause. So far only indirect evidence for their existence has been found (Plaschke et al., 2009c). The calculations in this paper reveal the structure of these modes under the stated limiting assumptions. Nevertheless, they yield testable relations for the velocity and the magnetic fields, which may help to identify in the future KSmodes in measurements obtained in the vicinity of the magnetopause and on the ground at cusp latitudes; more efforts in this direction are obviously necessary and in progress.

Acknowledgements. This work was financially supported by the German Ministerium für Wirtschaft und Technologie and the Deutsches Zentrum für Luft- und Raumfahrt under contract 50QP0402.

We gratefully acknowledge the participation in and discussions within the International Space Science Institute (ISSI) team "MultiScale Electrodynamics of Magnetosphere-Ionosphere Interactions at High Latitudes" coordinated by A. V. Streltsov.

Topical Editor R. Nakamura thanks S. Fujita and J. De Keyser for their help in evaluating this paper.

\section{References}

Allan, W. and Knox, F. B.: A dipole field model for axisymmetric Alfven waves with finite ionosphere conductivities, Planet. Space Sci., 27, 79-85, doi:10.1016/0032-0633(79)90149-1, 1979.

Allan, W. and Knox, F. B.: ULF pulsation mode coupling in the ionosphere and magnetosphere, Planet. Space Sci., 30, 285-292, doi:10.1016/0032-0633(82)90005-8, 1982.

Angelopoulos, V.: The THEMIS Mission, Space Sci. Rev., 141, 534, doi:10.1007/s11214-008-9336-1, 2008.

Aubry, M. P., Kivelson, M. G., and Russell, C. T.: Motion and structure of the magnetopause., J. Geophys. Res., 76, 1673-1696, doi:10.1029/JA076i007p01673, 1971.

Belmont, G., Reberac, F., and Rezeau, L.: Resonant amplification of magnetosheath MHD fluctuations at the magnetopause, Geophys. Res. Lett., 22, 295-298, doi:10.1029/94GL03078, 1995.

Berchem, J. and Russell, C. T.: The thickness of the magnetopause current layer - ISEE 1 and 2 observations, J. Geophys. Res., 87, 2108-2114, doi:10.1029/JA087iA04p02108, 1982.

Chen, L. and Hasegawa, A.: A Theory of Long-Period Magnetic Pulsations, 1. Steady State Excitation of Field Line Resonance, J. Geophys. Res., 79, 1024-1032, 1974a.

Chen, L. and Hasegawa, A.: A Theory of Long-Period Magnetic Pulsations, 2. Impulse Excitation of Surface Eigenmode, J. Geophys. Res., 79, 1033-1037, doi:10.1029/JA079i007p01033, 1974b.

Cummings, W. D., Sullivan, R. J., and Coleman, P. J.: Standing Alfvén Waves in the Magnetosphere, J. Geophys. Res., 74, 778793, doi:10.1029/JA074i003p00778, 1969.

De Keyser, J., Roth, M., Reberac, F., Rezeau, L., and Belmont, G.: Resonant amplification of MHD waves in realistic subsolar magnetopause configurations, J. Geophys. Res., 104, 23992410, doi:10.1029/1998JA900060, 1999.

De Keyser, J., Roth, M., Dunlop, M. W., Rème, H., Owen, C. J., and Paschmann, G.: Empirical reconstruction and longduration tracking of the magnetospheric boundary in singleand multi-spacecraft contexts, Ann. Geophys., 23, 1355-1369, doi:10.5194/angeo-23-1355-2005, 2005.

Fenrich, F. R., Samson, J. C., Sofko, G., and Greenwald, R. A.: ULF high- and low-m field line resonances observed with the Super Dual Auroral Radar Network, J. Geophys. Res., 100, 21535 21548, doi:10.1029/95JA02024, 1995.

Francia, P. and Villante, U.: Some evidence of ground power enhancements at frequencies of global magnetospheric modes at low latitude, Ann. Geophys., 15, 17-23, doi:10.1007/s00585997-0017-2, 1997.

Fujita, S., Glassmeier, K. H., and Kamide, K.: MHD waves generated by the Kelvin-Helmholtz instability in a nonuniform magnetosphere, J. Geophys. Res., 101, 27317-27326, doi:10.1029/96JA02676, 1996.

Glassmeier, K. and Heppner, C.: Traveling magnetospheric convection twin vortices - Another case study, global characteristics, and a model, J. Geophys. Res., 97, 3977-3992, doi:10.1029/91JA02464, 1992.

Glassmeier, K., Auster, H., Constantinescu, D., Fornaçon, K., Narita, Y., Plaschke, F., Angelopoulos, V., Georgescu, E., Baumjohann, W., Magnes, W., Nakamura, R., Carlson, C. W., Frey, S., McFadden, J. P., Phan, T., Mann, I., Rae, I. J., and Vogt, J.: Magnetospheric quasi-static response to the dynamic magnetosheath: A THEMIS case study, Geophys. Res. Lett., 35, 
L17S01, doi:10.1029/2008GL033469, 2008.

Haaland, S. E., Sonnerup, B. U. Ö., Dunlop, M. W., Balogh, A., Georgescu, E., Hasegawa, H., Klecker, B., Paschmann, G., Puhl-Quinn, P., Rème, H., Vaith, H., and Vaivads, A.: Fourspacecraft determination of magnetopause orientation, motion and thickness: comparison with results from single-spacecraft methods, Ann. Geophys., 22, 1347-1365, doi:10.5194/angeo22-1347-2004, 2004.

Kepko, L. and Spence, H. E.: Observations of discrete, global magnetospheric oscillations directly driven by solar wind density variations, J. Geophys. Res., 108, 1257-1269, doi:10.1029/2002JA009676, 2003.

Kepko, L., Spence, H. E., and Singer, H. J.: ULF waves in the solar wind as direct drivers of magnetospheric pulsations, Geophys. Res. Lett., 29, 1197-1200, doi:10.1029/2001GL014405, 2002.

Kruskal, M. and Schwarzschild, M.: Some Instabilities of a Completely Ionized Plasma, Proc. R. Soc. Lon., A223, 348-360, doi:10.1098/rspa.1954.0120, 1954.

Le, G. and Russell, C. T.: The thickness and structure of high beta magnetopause current layer, Geophys. Res. Lett., 21, 24512454, doi:10.1029/94GL02292, 1994.

Mathie, R. A., Mann, I. R., Menk, F. W., and Orr, D.: Pc5 ULF pulsations associated with waveguide modes observed with the IMAGE magnetometer array, J. Geophys. Res., 104, 7025-7036, doi:10.1029/1998JA900150, 1999.

Panov, E. V., Büchner, J., Fränz, M., Korth, A., Savin, S. P., Rème, H., and Fornaçon, K.: High-latitude Earth's magnetopause outside the cusp: Cluster observations, J. Geophys. Res., 113, A01220, doi:10.1029/2006JA012123, 2008.

Paschmann, G., Baumjohann, W., Sckopke, N., Sonnerup, B., and Papamastorakis, I.: The magnetopause and boundary layer for small magnetic shear - Convection electric fields and reconnection, Geophys. Res. Lett., 17, 1829-1832, doi:10.1029/GL017i011p01829, 1990.

Paschmann, G., Baumjohann, W., Sckopke, N., Phan, T., and Luehr, H.: Structure of the dayside magnetopause for low magnetic shear, J. Geophys. Res., 98, 13409-13422, doi:10.1029/93JA00646, 1993.

Paschmann, G., Haaland, S., Sonnerup, B. U. Ö., Hasegawa, H., Georgescu, E., Klecker, B., Phan, T. D., Rème, H., and Vaivads, A.: Characteristics of the near-tail dawn magnetopause and boundary layer, Ann. Geophys., 23, 1481-1497, doi:10.5194/angeo-23-1481-2005, 2005.

Phan, T. D. and Paschmann, G.: Low-latitude dayside magnetopause and boundary layer for high magnetic shear 1 . Structure and motion, J. Geophys. Res., 101, 7801-7816, doi:10.1029/95JA03752, 1996.

Phan, T., Paschmann, G., Baumjohann, W., Sckopke, N., and Luehr, H.: The magnetosheath region adjacent to the dayside magnetopause: AMPTE/IRM observations, J. Geophys. Res., 99, 121141, doi:10.1029/93JA02444, 1994.

Plaschke, F., Glassmeier, K., Auster, H. U., Angelopoulos, V., Constantinescu, O. D., Fornaçon, K., Georgescu, E., Magnes, W., McFadden, J. P., and Nakamura, R.: Statistical study of the magnetopause motion: First results from THEMIS, J. Geophys. Res., 114, A00C10, doi:10.1029/2008JA013423, 2009a.
Plaschke, F., Glassmeier, K., Auster, H. U., Constantinescu, O. D., Magnes, W., Angelopoulos, V., Sibeck, D. G., and McFadden, J. P.: Standing Alfvén waves at the magnetopause, Geophys. Res. Lett., 36, L02104, doi:10.1029/2008GL036411, 2009 b.

Plaschke, F., Glassmeier, K.-H., Sibeck, D. G., Auster, H. U., Constantinescu, O. D., Angelopoulos, V., and Magnes, W.: Magnetopause surface oscillation frequencies at different solar wind conditions, Ann. Geophys., 27, 4521-4532, doi:10.5194/angeo27-4521-2009, 2009c.

$\mathrm{Pu}, \mathrm{Z}$. and Kivelson, M. G.: Kelvin-Helmholtz instability at the magnetopause: Solution for compressible plasmas., J. Geophys. Res., 88, 841-852, doi:10.1029/JA088iA02p00841, 1983.

Ruohoniemi, J. M., Greenwald, R. A., Baker, K. B., and Samson, J. C.: HF radar observations of Pc 5 field line resonances in the midnight/early morning MLT sector, J. Geophys. Res., 96, 15697-15710, doi:10.1029/91JA00795, 1991.

Samson, J. C., Greenwald, R. A., Ruohoniemi, J. M., Hughes, T. J., and Wallis, D. D.: Magnetometer and radar observations of magnetohydrodynamic cavity modes in the Earth's magnetosphere, Can. J. Phys., 69, 929-937, doi:10.1139/p91-147, 1991.

Samson, J. C., Harrold, B. G., Ruohoniemi, J. M., Greenwald, R. A., and Walker, A. D. M.: Field line resonances associated with MHD waveguides in the magnetosphere, Geophys. Res. Lett., 19, 441-444, doi:10.1029/92GL00116, 1992.

Sarafopoulos, D. V.: A case study testing the cavity mode model of the magnetosphere, Ann. Geophys., 23, 1867-1880, doi:10.5194/angeo-23-1867-2005, 2005.

Shue, J. and Song, P.: The location and shape of the magnetopause, Planet. Space Sci., 50, 549-558, doi:10.1016/S00320633(02)00034-X, 2002.

Sonnerup, B. U. O. and Cahill, L. J.: Magnetopause structure and altitude from Explorer-12 observations, J. Geophys. Res., 72, 171-183, 1967.

Sonnerup, B. U. O. and Ledley, B. G.: Magnetopause rotational forms, J. Geophys. Res., 79, 4309-4314, doi:10.1029/JA079i028p04309, 1974.

Southwood, D. J.: Some features of field line resonances in the magnetosphere, Planet. Space Sci., 22, 483-491, doi:10.1016/0032-0633(74)90078-6, 1974.

Spreiter, J. R., Summers, A. L., and Alksne, A. Y.: Hydromagnetic flow around the magnetosphere, Planet. Space Sci., 14, 223-253, doi:10.1016/0032-0633(66)90124-3, 1966.

Uberoi, C.: Resonant absorption of Alfven compressional surface waves, J. Geophys. Res., 94, 6941-6944, doi:10.1029/JA094iA06p06941, 1989.

Walker, A. D. M.: The Kelvin-Helmholtz instability in the lowlatitude boundary layer, Planet. Space Sci., 29, 1119-1133, doi:10.1016/0032-0633(81)90011-8, 1981.

Ziesolleck, C. W. S. and McDiarmid, D. R.: Statistical survey of auroral latitude Pc 5 spectral and polarization characteristics, J. Geophys. Res., 100, 19299-19312, doi:10.1029/95JA00434, 1995. 